\title{
The full automorphism group of Cayley graphs of $\mathbb{Z}_{p} \times \mathbb{Z}_{p^{2}}$
}

\author{
Edward Dobson* \\ Department of Mathematics and Statistics \\ Mississippi State University \\ Mississippi State, MS 39762 U.S.A. \\ dobson@math.msstate.edu \\ and \\ University of Primorska \\ IAM \\ Muzejski trg 2 \\ 6000 Koper, Slovenia
}

Submitted: Oct 15, 2010; Accepted: Mar 5, 2012; Published: Mar 19, 2012

Mathematics Subject Classifications: 05C25, 20B25

\begin{abstract}
Let $p \geq 5$ be prime. We determine the full automorphism groups of Cayley digraphs of $\mathbb{Z}_{p} \times \mathbb{Z}_{p^{2}}$.
\end{abstract}

\section{Introduction}

Determining the full automorphism group of a Cayley digraph of a group $G$ is perhaps one of the most fundamental questions that one can ask about a Cayley digraph, and seems to be a very difficult question to answer. In recent years, progress towards solving this problem has begun, usually focusing on Cayley digraphs of specific groups $G$ or Cayley digraphs that have particular properties, such Cayley digraphs that $1 / 2$-transitive, or edge-transitive. The groups $G$ for which the full automorphism groups of Cayley digraphs of $G$ have been explicitly determined are $G=\mathbb{Z}_{p}[1], \mathbb{Z}_{p}^{2}[15], \mathbb{Z}_{p^{2}}[18]$ (see [15] for a later proof), $\mathbb{Z}_{p q}$ [18] (see [10] for a later proof), the nonabelian groups of order $p q[10]$, and $\mathbb{Z}_{p}^{3}[12]$, where $p$ and $q$ are distinct primes. Additionally, strong constraints on the structure of the full automorphism group of Cayley digraphs of $\mathbb{Z}_{n}$ have been obtained (see [20]), and independently for $n$ square-free [13]. Using these constraints, Ponomarenko [22] has

${ }^{*}$ Project sponsored by the National Security Agency under Grant Number H98230-11-1-0179 
found a polynomial time algorithm to compute the full automorphism group of circulant digraphs. In this paper, we determine the full automorphism groups of Cayley digraphs of $\mathbb{Z}_{p} \times \mathbb{Z}_{p^{2}}, p \geq 5$. Our approach basically follows the approach used to determine the full automorphism groups of Cayley digraphs of $\mathbb{Z}_{p}^{3}$ given in [12]. We use the implicit determination of all Sylow $p$-subgroups of Cayley digraphs of $\mathbb{Z}_{p} \times \mathbb{Z}_{p^{2}}$ given in [8], and then either determine the overgroups of these $p$-subgroups or use known results giving the overgroups of these $p$-groups.

For permutation group terms not defined here, see [6]. We begin with some definitions, and then state some of the many results in the literature that we will have need of.

Definition 1 Let $G$ be a group and $S \subset G$ such that $1_{G} \notin S$. Define a digraph $D=$ $D(G, S)$ by $V(D)=G$ and $E(D)=\left\{(u, v): v^{-1} u \in S\right\}$. Such a digraph is a Cayley digraph of $G$ with connection set $S$. A Cayley graph of $G$ is defined analogously although we insist that $S=S^{-1}=\left\{s^{-1}: s \in S\right\}$. A circulant (di)graph of order $n$ is simply a Cayley (di)graph of $\mathbb{Z}_{n}$.

It is straightforward to verify that for $g \in G$, the map $g_{L}: G \rightarrow G$ by $g_{L}(x)=g x$ is an automorphism of a Cayley digraph $D$ of a group $G$. Thus $G_{L}=\left\{g_{L}: g \in G\right\}$, the left regular representation of $G$, is a subgroup of the automorphism group of $D, \operatorname{Aut}(D)$. Sabidussi has shown [23] that a digraph $D$ is isomorphic to a Cayley digraph of $D$ if and only if $\operatorname{Aut}(D)$ contains a regular subgroup isomorphic to $G$.

Definition 2 Let $G$ be a transitive permutation group with complete block system $\mathcal{B}$. We say that $\mathcal{B}$ is genuine if $\mathcal{B}$ is formed by the orbits of some normal subgroup of $G$. By $G / \mathcal{B}$, we mean the subgroup of $S_{\mathcal{B}}$ induced by the action of $G$ on $\mathcal{B}$, and by $\operatorname{fix}_{G}(\mathcal{B})$ the kernel of this action. Thus $\operatorname{fix}_{G}(\mathcal{B})=\{g \in G: g(B)=B$ for all $B \in \mathcal{B}\}$. By $\operatorname{Stab}_{G}(\mathcal{B})$, we mean the set-wise stabilizer in $G$ of the block $B \in \mathcal{B}$. Hence $\operatorname{Stab}_{G}(B)=\{g \in G: g(B)=B\}$, and $\operatorname{fix}_{G}(\mathcal{B})=\cap_{B \in \mathcal{B}} \operatorname{Stab}_{G}(B)$. If $\mathcal{C}$ is a complete block system of $G$ such that every block of $\mathcal{C}$ is a union of blocks of $\mathcal{B}$, we write $\mathcal{B} \preceq \mathcal{C}$, and denote the complete block system of $G / \mathcal{B}$ induced by $\mathcal{C}$ by $\mathcal{C} / \mathcal{B}$. Thus $C / \mathcal{B} \in \mathcal{C} / \mathcal{B}$ consists of those blocks of $\mathcal{B}$ whose union is $C \in \mathcal{C}$.

In most situations, we will be determining all 2-closed groups, which are a slightly larger class of groups than automorphism groups of digraphs, and are defined below.

Definition 3 Let $\Omega$ be a set and $G \leq S_{\Omega}$. Let $G$ act on $\Omega \times \Omega$ by $g\left(\omega_{1}, \omega_{2}\right)=\left(g\left(\omega_{1}\right), g\left(\omega_{2}\right)\right)$ for every $g \in G$ and $\omega_{1}, \omega_{2} \in \Omega$. We define the 2 -closure of $G$, denoted $G{ }^{(2)}$, to be the largest subgroup of $S_{\Omega}$ whose orbits on $\Omega \times \Omega$ are the same as $G$ 's. Let $\mathcal{O}_{1}, \ldots, \mathcal{O}_{r}$ be the orbits of $G$ acting on $\Omega \times \Omega$. Define digraphs $\Gamma_{1}, \ldots, \Gamma_{r}$ by $V\left(\Gamma_{i}\right)=\Omega$ and $E\left(\Gamma_{i}\right)=\mathcal{O}_{i}$. Each $\Gamma_{i}, 1 \leq i \leq r$, is an orbital digraph of $G$, and it is straightforward to show that $G^{(2)}=\cap_{i=1}^{r} \operatorname{Aut}\left(\Gamma_{i}\right)$. Equivalently, $G^{(2)}$ is the automorphism group of a color digraph.

Definition 4 For a positive integer $n$, define $N(n)=\left\{x \rightarrow a x+b: a \in \mathbb{Z}_{n}^{*}, b \in \mathbb{Z}_{n}\right\}$. Thus $N(n)$ is the normalizer of the left regular representation $\left(\mathbb{Z}_{n}\right)_{L}$ of $\mathbb{Z}_{n}$ in $S_{n}$. We remark that for $p$ a prime, $N(p)$ is usually denoted $\mathrm{AGL}(1, p)$. 
The following classical result of Burnside [2] is quite useful for analyzing transitive groups of prime degree, especially now that, as a consequence of the Classification of Finite Simple Groups, all doubly transitive groups are known [3]. We remark that the following versions of this result also makes use another of Burnside's results, namely [6, Theorem 4.1B].

Theorem 5 Let $G$ be a transitive group of prime degree. Then either $G$ is doubly transitive with nonabelian simple socle, or $G$ contains a normal Sylow p-subgroup.

Equivalently (see [6, Exercise 3.5.1]), we have

Theorem 6 Let $G$ be a transitive group of prime degree $p$. Then we may relabel the set upon which $G$ acts so that $G \leq \operatorname{AGL}(1, p)$, or $G$ is doubly transitive with nonabelian simple socle.

The following result is [9, Theorem 33], and is an extension of the previous result to prime-powers.

Theorem 7 Let $p \geq 3$ be prime, and $G \leq S_{p^{m}}, m \geq 1$, be transitive such that every minimal transitive subgroup of $G$ is cyclic. Then either $G$ contains a transitive normal Sylow p-subgroup, or $G$ is doubly-transitive and

1. $G=A_{p^{m}}$ or $S_{p^{m}}$, and $m=1$,

2. $\operatorname{PSL}(n, k) \leq G \leq \operatorname{P\Gamma L}(n, k)$, for some prime power $k$ and $n \geq 2$ with $p^{m}=\left(k^{n}-\right.$ $1) /(k-1)$,

3. $\operatorname{PSL}(2,11)$ or $M_{11}$ and $p^{m}=11$,

4. $M_{23}$ and $p^{m}=23$.

The following is [9, Lemma 17], and gives more information concerning some of the doubly-transitive groups of the preceding result.

Lemma 8 Let $\operatorname{PSL}(n, k) \leq G \leq \operatorname{P\Gamma L}(n, k)$ be primitive of degree $\left(k^{n}-1\right) /(k-1)=p^{m}$, where $k$ is a prime power, $n \geq 2, p$ an odd prime, and $m \geq 1$. If $(n, k) \neq(2,8)$, then a Sylow p-subgroup of $\operatorname{P\Gamma L}(n, k)$ is regular and cyclic, and so a Sylow p-subgroup of $G$ is regular and cyclic. Consequently, if $N\left(p^{m}\right) \leq G, m \geq 2$, then $p^{m}=9$ and $\operatorname{PSL}\left(2,2^{3}\right)<G \leq \operatorname{P\Gamma L}\left(2,2^{3}\right)$.

The following definition and result are very useful in determining Sylow $p$-subgroups of automorphism groups of Cayley digraphs of prime-power order (among other things).

Definition 9 Let $G$ be a transitive permutation group that admits a complete block system $\mathcal{B}$ of $m$ blocks of size $p, p$ a prime, and $\mathcal{B}$ is formed by the orbits of some normal subgroup $N \triangleleft G$. Then for each $B \in \mathcal{B}$ there exists $\alpha_{B} \in N$ such that $\left.\alpha_{B}\right|_{B}$ is a p-cycle. Define an equivalence relation $\equiv$ on the blocks of $\mathcal{B}$ by $B \equiv B^{\prime}$ if and only if whenever $\alpha \in N$ then $\left.\alpha\right|_{B}$ is a p-cycle if and only if $\left.\alpha\right|_{B^{\prime}}$ is also a p-cycle. Denote the equivalence classes of $\equiv$ by $C_{0}, \ldots, C_{a}$ and let $E_{i}=\cup_{B \in C_{i}} B$. 
The following result is [7, Lemma 3].

Lemma 10 Let $G$ be as in Definition 9, and $\alpha \in N$ be such that $|\alpha|=p$. Then for each $0 \leq i \leq a$ there exists $\alpha_{i} \in G^{(2)}$ such that $\left.\alpha_{i}\right|_{E_{i}}=\left.\alpha\right|_{E_{i}}$ and $\left.\alpha_{i}\right|_{E_{j}}=1$ for every $i \neq j$. Furthermore, each $E_{i}$ is a block of $G$.

We remark that the statement of Lemma 10 is more general than in [9], but it is straightforward to show this more general version holds using the fact that the 2-closure of $G$ is the intersection of the automorphism groups of the orbital digraphs of $G$.

We shall have need of the following result of Kalužnin and Klin [17] (this result is also contained in the more easily accessible [4, Theorem 5.1]).

Lemma 11 Let $G \leq S_{X}$ and $H \leq S_{Y}$ be transitive groups. Then in their coordinate-wise action on $X \times Y$, we have

$$
(G \times H)^{(2)}=G^{(2)} \times H^{(2)} \text {, and }(G \curlywedge H)^{(2)}=G^{(2)} \prec H^{(2)} .
$$

For the remainder of this paper, we define $\tau_{1}, \tau_{2}: \mathbb{Z}_{p} \times \mathbb{Z}_{p^{2}} \rightarrow \mathbb{Z}_{p} \times \mathbb{Z}_{p^{2}}$ by $\tau_{1}(i, j)=$ $(i+1, j)$ and $\tau_{2}(i, j)=(i, j+1)$. Then $\left\langle\tau_{1}, \tau_{2}\right\rangle=\left(\mathbb{Z}_{p} \times \mathbb{Z}_{p^{2}}\right)_{L}$ and so $\left\langle\tau_{1}, \tau_{2}\right\rangle \leq \operatorname{Aut}(\Gamma)$ for every Cayley digraph $\Gamma$ of $\mathbb{Z}_{p} \times \mathbb{Z}_{p^{2}}$.

The following result can be extracted from [8, Theorem 10], together with the previous result, and gives the Sylow $p$-subgroups of the automorphism groups of Cayley digraphs of $\mathbb{Z}_{p} \times \mathbb{Z}_{p^{2}}$. Let $\mathcal{B}_{1,1}$ be the complete block system of $\left\langle\tau_{1}, \tau_{2}\right\rangle$ formed by the orbits of $\left\langle\tau_{1}, \tau_{2}^{p}\right\rangle$, and $\mathcal{B}_{2}$ the complete block system of $\left\langle\tau_{1}, \tau_{2}\right\rangle$ formed by the orbits of $\left\langle\tau_{2}\right\rangle$. In the following result, by $\left.\gamma\right|_{B}$ we mean the permutation of $\mathbb{Z}_{p} \times \mathbb{Z}_{p^{2}}$ defined by $\left.\gamma\right|_{B}(x)=\gamma(x)$ if $x \in B$, and $\left.\gamma\right|_{B}(x)=x$ if $x \notin B$.

Theorem 12 Let $H \leq S_{\mathbb{Z}_{p} \times \mathbb{Z}_{p^{2}}}$ be 2-closed with Sylow p-subgroup $P$ which contains $\left(\mathbb{Z}_{p} \times\right.$ $\left.\mathbb{Z}_{p^{2}}\right)_{L}$. Then one of the following is true for some $\alpha_{1} \in \operatorname{Aut}\left(\mathbb{Z}_{p} \times \mathbb{Z}_{p^{2}}\right)_{L}$ :

(i) $H=S_{\mathbb{Z}_{p} \times \mathbb{Z}_{p^{2}}}$,

(ii) $P=\left(\mathbb{Z}_{p} \times \mathbb{Z}_{p^{2}}\right)_{L}$,

(iii) $P=\alpha_{1}^{-1}\left\langle\tau_{1}, \tau_{2},\left.\tau_{2}^{p}\right|_{B}: B \in \mathcal{B}_{1,1}\right\rangle \alpha_{1} \cong \mathbb{Z}_{p} \times\left(\mathbb{Z}_{p}\left\langle\mathbb{Z}_{p}\right)\right.$,

(iv) $P=\alpha_{1}^{-1}\left\langle\tau_{1}, \tau_{2},\left.\tau_{2}^{p}\right|_{B}: B \in \mathcal{B}_{2}\right\rangle \alpha_{1}$,

(v) $P=\alpha_{1}^{-1}\left\langle\tau_{1}, \tau_{2},\left.\tau_{1}\right|_{B}: B \in \mathcal{B}_{1,1}\right\rangle \alpha_{1}$,

(vi) if $\gamma: \mathbb{Z}_{p} \times \mathbb{Z}_{p^{2}} \rightarrow \mathbb{Z}_{p} \times \mathbb{Z}_{p^{2}}$ by $\gamma(i, j)=(i+[a j(\bmod p)], j+i b p), a, b \in \mathbb{Z}_{p}^{*}$, then $P=\left\langle\tau_{1}, \tau_{2}, \gamma\right\rangle$, and $|P|=p^{4}$,

(vii) $\alpha_{1}^{-1} P \alpha_{1}=P_{1} 2 P_{2}$, where $P_{1}$ is 2 -closed $p$-group of degree $p^{2}$ and contains a regular subgroup isomorphic to $\mathbb{Z}_{p^{2}}$ or $\mathbb{Z}_{p}^{2}$, and $P_{2} \leq S_{p}$ is cyclic of order $p$,

(viii) $\alpha_{1}^{-1} P \alpha_{1}=P_{2} \prec P_{1}$, where $P_{2} \leq S_{p}$ is cyclic of order $p$, and $P_{1} \leq S_{p^{2}}$ is 2-closed p-subgroup of degree $p^{2}$ and contains a regular subgroup isomorphic to $\mathbb{Z}_{p^{2}}$. 
We remark that in $[8$, Theorem 10], there is an additional case, namely when $P$ admits a complete block system $\mathcal{B}^{\prime}$ consisting of $p^{2}$ blocks of size $p$ formed by the orbits of $\left\langle\tau_{1}\right\rangle$, $\operatorname{fix}_{P}\left(\mathcal{B}^{\prime}\right)=\left\langle\tau_{1}\right\rangle, P$ admits $\mathcal{B}_{1,1}$ as a complete block system, $\left.\operatorname{fix}_{P}\left(\mathcal{B}_{1,1}\right)\right|_{B} \not \leq\left.\left\langle\tau_{1}, \tau_{2}^{p}\right\rangle\right|_{B}$ for some $B \in \mathcal{B}_{1,1}$, and $\left\langle\tau_{1}, \tau_{2}\right\rangle \triangleleft P$. This case is superfluous, as if $\left\langle\tau_{1}, \tau_{2}\right\rangle \triangleleft P$, then $P$ admits a complete block system formed by the orbits of $\left\langle\tau_{2}^{p}\right\rangle$. This follows as if $\left\langle\tau_{1}, \tau_{2}\right\rangle \triangleleft P$, then $\gamma^{-1}\left\langle\tau_{2}\right\rangle \gamma=\left\langle\tau_{2} \tau_{1}^{a}\right\rangle$ for some $a \in \mathbb{Z}_{p}$, and so $\gamma^{-1}\left\langle\tau_{2}^{p}\right\rangle \gamma=\left\langle\tau_{2}^{p}\right\rangle$. Thus $\left\langle\tau_{2}^{p}\right\rangle \triangleleft P$ and its orbits form a complete block system (which is a case considered separately in [8, Theorem 10].

We shall also have need of the following result [11, Corollary 7.3], which gives the 2-closed groups which contain a regular abelian Sylow $p$-subgroup that is of rank 2 (i.e. is a direct product of two cyclic groups).

Theorem 13 Let $G \leq S_{p^{k}}$ be transitive and 2-closed with Sylow p-subgroup $P$ that is abelian of rank two. Then one of the following is true:

1. G has a normal Sylow p-subgroup,

2. $G$ is primitive, $k=2$, and $G$ is permutation isomorphic to $S_{2} \prec S_{p}$,

3. $k=2$, and $G$ is permutation isomorphic to $S_{p} \times S_{p}$, or

4. $G$ is permutation isomorphic to $S_{p} \times A$, where $A \leq N\left(p^{k-1}\right)$ has order dividing $(p-1) p^{k-1}$.

The following result deals with the case where a Sylow $p$-subgroup $P$ of a 2-closed group is permutation isomorphic to $\mathbb{Z}_{p} \times\left(\mathbb{Z}_{p} \prec \mathbb{Z}_{p}\right)$ and is extracted from $[12$, Proposition 5.12]. We remark that $\mathbb{Z}_{p} \times\left(\mathbb{Z}_{p} \prec \mathbb{Z}_{p}\right)$ contains regular subgroups isomorphic to both $\mathbb{Z}_{p} \times \mathbb{Z}_{p^{2}}$ and to $\mathbb{Z}_{p} \times \mathbb{Z}_{p} \times \mathbb{Z}_{p}$ as $\mathbb{Z}_{p}\left\langle\mathbb{Z}_{p}\right.$ contains regular subgroups isomorphic to $\mathbb{Z}_{p^{2}}$ and $\mathbb{Z}_{p} \times \mathbb{Z}_{p}[15$, Lemma 4$]$, and so any Cayley digraph of $\mathbb{Z}_{p} \times \mathbb{Z}_{p^{2}}$ that contains a Sylow $p$-subgroup permutation isomorphic to $\mathbb{Z}_{p} \times\left(\mathbb{Z}_{p}\left(\mathbb{Z}_{p}\right)\right.$ is also isomorphic to a Cayley digraph of $\mathbb{Z}_{p} \times \mathbb{Z}_{p} \times \mathbb{Z}_{p}$.

Lemma 14 Let $H \leq S_{\mathbb{Z}_{p} \times \mathbb{Z}_{p^{2}}}$ be such that $\mathbb{Z}_{p} \times\left(\mathbb{Z}_{p} \imath \mathbb{Z}_{p}\right)$. Then $H$ is permutation isomorphic to $X \times S_{p^{2}}$ or $C((X \prec Y) \times Z)$, where $X, Y, Z \leq S_{p}$ are 2 -closed and $C \leq \operatorname{Aut}\left(\mathbb{Z}_{p}^{3}\right)$.

The following fact is quite well-known, and will be used implicitly throughout this paper. It is stated and proved here for convenience and conpleteness.

Lemma 15 Let $G$ be a regular abelian group of order $n$ and $H \leq S_{n}$ such that $G \leq H$. Then every complete block system of $H$ is genuine and is also formed by the orbits of some subgroup of $G$.

Proof. Let $\mathcal{B}$ be a complete block system of $H$ consisting of, say, $m$ blocks of size $k$. We will show that $\operatorname{fix}_{H}(\mathcal{B})$ contains a subgroup of $G$ of order $k$. Indeed, $G / \mathcal{B}$ is transitive and abelian, and as a transitive abelian group is regular [24, Proposition 4.3], $|G / \mathcal{B}|=m$, and so $\operatorname{fix}_{G}(\mathcal{B})$ has order $k$. Then $\mathcal{B}$ is formed by the orbits of $\operatorname{fix}_{G}(\mathcal{B})$, and so of $\operatorname{fix}_{H}(\mathcal{B}) \triangleleft H$.

The following is essentially a variant of [12, Lemma 2.8]. 
Lemma 16 Let $G$ be a group and $H \leq S_{G}$ such that $G_{L} \leq H$. Suppose that $G_{L} \leq K \triangleleft H$ and any two regular subgroups of $K$ isomorphic to $G$ are conjugate in $K$. Then $H \leq$ $\operatorname{Aut}(G) \cdot K$.

Proof. Let $h \in H$, so that $h^{-1} K h=K$ and $h^{-1} G_{L} h \leq K$ is a regular subgroup isomorphic to $G$. By hypothesis, there exists $k \in K$ such that $k^{-1} h^{-1} G_{L} h k=G_{L}$. By [6, Corollary 4.2B], we then have that $h k \in \operatorname{Aut}(G) \cdot G_{L}$, so that $h k=\alpha g_{L}, \alpha \in \operatorname{Aut}(G)$ and $g \in G$. As $k \in K$, we have that $h=\alpha\left(g_{L} k^{-1}\right)$ and $g_{L} k^{-1} \in K$, so the result follows.

\section{Overgroups of some 2-closed $p$-subgroups}

Our goal in this section is to find the overgroups of the 2-closed $p$-groups given in Theorem 12 parts (iv), (v), and (vi), and when the overgroups do not normalize some natural $p$ subgroup, determine all such 2-closed overgroups.

The overgroups for Theorem 12 (iv) are given in Lemma 21, overgroups for Theorem 12 (v) are given in Lemma 25, while such 2-closed overgroups are given in Lemma 26. It is then shown that every group with Sylow $p$-subgroup as in Theorem 12 (vi) normalizes $\left\langle\tau_{1}, \tau_{2}\right\rangle=\left(\mathbb{Z}_{p} \times \mathbb{Z}_{p^{2}}\right)_{L}$ in Lemma 30 . We begin with a technical lemma.

Lemma 17 Let $H \leq S_{n \cdot m}, m \geq 4$, admit a complete block system $\mathcal{D}$ with blocks of size m. If $\left.\operatorname{fix}_{H}(\mathcal{D})\right|_{D} \geq A_{m}$ and $\operatorname{fix}_{H}(\mathcal{D})$ acts faithfully on $D \in \mathcal{D}$, then $H \leq S_{n} \times S_{m}$ or $m=6$.

Proof. As $\left.\operatorname{fix}_{H}(\mathcal{D})\right|_{D} \geq A_{m}$ is primitive, $\operatorname{Stab}_{\left.\mathrm{fix}_{H}(\mathcal{D})\right|_{D}}(d), d \in D$, fixes exactly one point. If $m=4$, then $A_{4}$ has a unique subgroup of order 4 , and so by the comments following [6, Lemma $1.6 \mathrm{~B}], A_{4}$ has a unique transitive representation of degree 4 . Thus $\left.\operatorname{fix}_{H}(\mathcal{D})\right|_{D}$ is equivalent to $\left.\operatorname{fix}_{H}(\mathcal{D})\right|_{D^{\prime}}$ for all $D, D^{\prime} \in \mathcal{D}$. If $m \geq 5, m \neq 6$, then by [3, Table], we have that $\left.\operatorname{fix}_{H}(\mathcal{D})\right|_{D}$ is equivalent to $\left.\operatorname{fix}_{H}(\mathcal{D})\right|_{D^{\prime}}$ for every $D, D^{\prime} \in \mathcal{D}$. The result then follows by [11, Lemma 4.1].

For the following result, let $P_{1}=\left\langle\tau_{1}, \tau_{2},\left.\tau_{1}\right|_{B}: B \in \mathcal{B}_{1,1}\right\rangle$ and $P_{2}=\left\langle\tau_{1}, \tau_{2},\left.\tau_{2}^{p}\right|_{B}: B \in\right.$ $\left.\mathcal{B}_{2}\right\rangle$. Also, we define a minimal complete block system $\mathcal{B}$ of a transitive group $H$ to be a complete block system of $H$ such that there is no nontrivial complete block system $\mathcal{C} \prec \mathcal{B}$.

Lemma 18 Let $H \leq S_{\mathbb{Z}_{p} \times \mathbb{Z}_{p^{2}}}, p \geq 3$, have Sylow p-subgroup $P=P_{1}$ or $P_{2}$. Then $H$ is imprimitive, and if $\mathcal{D}$ is a minimal complete block system of $H$, then $\mathcal{D}$ has blocks of prime size.

Proof. By [24, Theorem 25.5], $H$ is imprimitive or doubly-transitive. The primitive groups that contain a transitive abelian subgroup are given in [19, Theorem 1.1], while the doubly-transitive groups are given in [3, Table]. We conclude that $H$ is imprimitive. Let $\mathcal{D}$ be a minimal complete block system of $H$. We assume that $\mathcal{D}$ consists of $p$ blocks of size $p^{2}$. By $\left[6\right.$, Exercise 1.5.10], we have that $\left.\operatorname{Stab}_{H}(D)\right|_{D}$ is primitive for $D \in \mathcal{D}$. As 
$H / \mathcal{D} \leq S_{p}$, we have that a Sylow $p$-subgroup of $\operatorname{fix}_{H}(\mathcal{D})$ has order $p^{p+1}$ as $P$ has order $p^{p+2}$.

Suppose that $\operatorname{fix}_{H}(\mathcal{D})$ acts faithfully on $D \in \mathcal{D}$. Then a Sylow $p$-subgroup of $\left.\operatorname{fix}_{H}(\mathcal{D})\right|_{D}$ has order $p^{p+1}$ and degree $p^{2}$, and so must be $\mathbb{Z}_{p} \prec \mathbb{Z}_{p}$. Perusing the list of primitive groups that contain a transitive abelian subgroup in $\left[19\right.$, Theorem 1.1], we see that $\left.\operatorname{Stab}_{H}(\mathcal{D})\right|_{D} \geq$ $A_{p^{2}}$. As $p \geq 3, A_{p^{2}}$ is simple, and so $\left.\operatorname{fix}_{H}(\mathcal{D})\right|_{D} \geq A_{p^{2}}$. It then follows by Lemma 17 that $H$ is canonically isomorphic to a subgroup of $S_{p} \times S_{p^{2}}$, and so has Sylow $p$-subgroup isomorphic to $\mathbb{Z}_{p} \times\left(\mathbb{Z}_{p}\left\langle\mathbb{Z}_{p}\right)\right.$. However, $P$ is not isomorphic to $\mathbb{Z}_{p} \times\left(\mathbb{Z}_{p}\left\langle\mathbb{Z}_{p}\right)\right.$, a contradiction. We henceforth assume that $\operatorname{fix}_{H}(\mathcal{D})$ acts unfaithfully on $D \in \mathcal{D}$.

As $\mathcal{D}$ is genuine, $\mathcal{D}$ is formed by the orbits of some subgroup of $\left\langle\tau_{1}, \tau_{2}\right\rangle$ of order $p^{2}$, and so $\mathcal{D}$ is formed by the orbits of $\left\langle\tau_{1}^{a} \tau_{2}\right\rangle$ or $\left\langle\tau_{1}, \tau_{2}^{p}\right\rangle$ for some $a \in \mathbb{Z}_{p}$. Then $\left\langle\tau_{2}^{p}\right\rangle$ is contained in every subgroup of $\left\langle\tau_{1}, \tau_{2}\right\rangle$ of order $p^{2}$. Let $\mathcal{D}_{1}$ and $\mathcal{D}_{2}$ be two distinct complete block systems of $\left\langle\tau_{1}, \tau_{2}\right\rangle$ with blocks of size $p^{2}$. Considering intersections of blocks chosen from $\mathcal{D}_{1}$ and $\mathcal{D}_{2}$, we see that each such intersection has at most $p$ elements contained in it, every element of $\mathbb{Z}_{p} \times \mathbb{Z}_{p^{2}}$ is contained in such an intersection, and there are at most $p^{2}$ such intersections. We conclude that each such intersection has order exactly $p$ (and as $\left\langle\tau_{2}^{p}\right\rangle \leq \operatorname{fix}_{H}\left(\mathcal{D}_{1}\right) \cap \operatorname{fix}_{H}\left(\mathcal{D}_{2}\right)$ each such intersection is an orbit of $\left.\left\langle\tau_{2}^{p}\right\rangle\right)$.

Let $D \in \mathcal{D}$. Then for some $D^{\prime} \neq D, D^{\prime} \in \mathcal{D}$, there exists $\gamma \in \operatorname{fix}_{H}(\mathcal{D})$ such that $\left.\gamma\right|_{D}=1$ but $\left.\gamma\right|_{D^{\prime}} \neq 1$. Then $\langle\gamma\rangle^{\text {fix }_{H}(\mathcal{D})}$, the normal closure of $\langle\gamma\rangle$ in $\operatorname{fix}_{H}(\mathcal{D})$, is normal in $\operatorname{fix}_{H}(\mathcal{D})$, so the orbits of $\left.\langle\gamma\rangle^{\mathrm{fix}_{H}(\mathcal{D})}\right|_{D^{\prime}}$ form a complete block system of $\left.\operatorname{fix}_{H}(\mathcal{D})\right|_{D^{\prime}}$, and so have order $p$ or $p^{2}$. We conclude that some element $\delta$ of $\operatorname{fix}_{H}(\mathcal{D})$ that is the identity on $D$ has order $p$ on $D^{\prime}$. After an appropriate conjugation by an element of $\operatorname{fix}_{H}(\mathcal{D})$, if necessary, we may assume that this element is in $P$. If $P=P_{1}$ then the only nontrivial elements that fix a point are contained in $\left\langle\left.\tau_{1}\right|_{B}: B \in \mathcal{B}_{1,1}\right\rangle$, while if $P=P_{2}$ then the only nontrivial elements that fix a point are contained in $\left\langle\left.\tau_{2}^{p}\right|_{B}: B \in \mathcal{B}_{2}\right\rangle$. In the former case, let $B \in \mathcal{B}_{1,1}$ such that $\left.\delta\right|_{B}$ is semiregular of order $p$ while in the latter case let $B \in \mathcal{B}_{2}$ such that $\left.\delta\right|_{B}$ is semiregular of order $p$. If $P=P_{1}$ and $\mathcal{D} \neq \mathcal{B}_{1,1}$ or $P=P_{2}$ and $\mathcal{D} \neq \mathcal{B}_{2}$, then by arguments in the preceding paragraph $|D \cap B|=p$ for every $B \in \mathcal{B}_{1,1}$ if $P=P_{1}$ or $B \in \mathcal{B}_{2}$ if $P=P_{2}$. We conclude that $\delta$ is not the identity on any block of $\mathcal{D}$, a contradiction. Thus if $P=P_{1}$ then $\mathcal{D}=\mathcal{B}_{1,1}$ while if $P=P_{2}$ then $\mathcal{D}=\mathcal{B}_{2}$.

As $P=P_{1}$ and $\mathcal{D}=\mathcal{B}_{1,1}$ or $P=P_{2}$ and $\mathcal{D}=\mathcal{B}_{2}$, for some element $\tau \in\left\langle\tau_{1}, \tau_{2}\right\rangle$ we have that $\left.\tau\right|_{D^{\prime}} \in P\left(\tau=\tau_{1}\right.$ if $P=P_{1}$ while $\tau=\tau_{2}^{p}$ if $\left.P=P_{2}\right)$ for some $D^{\prime} \in \mathcal{D}$. Then $\left.\left.\tau\right|_{D} \in \operatorname{fix}_{H}(\mathcal{D})\right|_{D^{\prime}}$ for every $D \in \mathcal{D}$. As a normal subgroup of a primitive group is transitive [24, Theorem 8.8], the normal closure of $\left\langle\left.\tau\right|_{D}\right\rangle$ in $\operatorname{fix}_{H}(\mathcal{D})$ is transitive on $D$ and the identity on any block of $\mathcal{D}$ not $D$. We conclude that the order of $\operatorname{fix}_{H}(\mathcal{D})$ is divisible by $\left(p^{2}\right)^{p}$, and so $2 p=p+1$ or $p=1$, a contradiction.

Definition 19 For a permutation group $H \leq S_{\Omega}$, we define the support of $H$, denoted $\operatorname{supp}(H)$, to be the set of all $x \in \Omega$ such that there exists $h \in H$ such that $h(x) \neq x$.

Lemma 20 Let $H \leq S_{\mathbb{Z}_{p} \times \mathbb{Z}_{p^{2}}}$ have Sylow p-subgroup $P=\left\langle\tau_{1}, \tau_{2},\left.\tau_{2}^{p}\right|_{B}: B \in \mathcal{B}_{2}\right\rangle$. Then $\beta H \beta^{-1} \leq\left\{(i, j) \mapsto\left(\omega(i), \alpha j+a+p b_{i}\right): \omega \in S_{p}, \alpha \in \mathbb{Z}_{p^{2}}^{*}, a, b_{i} \in \mathbb{Z}_{p}\right\}$ for some $\beta \in$ $\operatorname{Aut}\left(\mathbb{Z}_{p} \times \mathbb{Z}_{p^{2}}\right)$. 
Proof. By Lemma 18 we have that $H$ admits a genuine complete block system $\mathcal{D}$ with blocks of prime size formed by the orbits of some subgroup $K$ of $\left\langle\tau_{1}, \tau_{2}\right\rangle$. Thus $K=\left\langle\tau_{2}^{p}\right\rangle$ or $\left\langle\tau_{1} \tau_{2}^{a p}\right\rangle$, where $a \in \mathbb{Z}_{p}$. As $\left.\tau_{2}\right|_{B} \in P$ for every $B \in \mathcal{B}_{2}$, it cannot be the case that $K=\left\langle\tau_{1} \tau_{2}^{a p}\right\rangle$ (as this would imply that $\operatorname{fix}_{P}(\mathcal{D})=\left\langle\tau_{1} \tau_{2}^{a p}\right\rangle \triangleleft P$, which is not true), so that $\mathcal{D}$ is formed by the orbits of $\left\langle\tau_{2}^{p}\right\rangle$. Observe that $\left\langle\left.\tau_{2}^{p}\right|_{B}: B \in \mathcal{B}_{2}\right\rangle$ is a Sylow $p$-subgroup of fix ${ }_{H}(\mathcal{D})$, so if $h \in H$ there exists $g \in$ fix $_{H}(\mathcal{D})$ such that $(h g)^{-1}\left\langle\left.\tau_{2}^{p}\right|_{B}: B \in \mathcal{B}_{2}\right\rangle(h g)=$ $\left\langle\left.\tau_{2}^{p}\right|_{B}: B \in \mathcal{B}_{2}\right\rangle$. As $g$ fixes each block of $\mathcal{D}$ and $\mathcal{D} \prec \mathcal{B}_{2}$, we have that $g$ fixes each block of $\mathcal{B}_{2}$. Now, if $B \in \mathcal{B}_{2}$ and $h \in H$, then $\left.(h g)^{-1} \tau_{2}^{p}\right|_{B}(h g)=\left.\tau_{2}^{x p}\right|_{B^{\prime}}$ for some $B^{\prime} \in \mathcal{B}_{2}$ and $x \in \mathbb{Z}_{p}^{*}$. We conclude that $h g$ maps the support of $\left.\tau_{2}^{p}\right|_{B}$ to the support of $\left.\tau_{2}^{p}\right|_{B^{\prime}}$, and so $h g(B)=B^{\prime}$. As $g(B)=B, h(B)=B^{\prime}$. Thus $\mathcal{B}_{2}$ is a complete block system of $H$ as well.

Now, a Sylow $p$-subgroup of $\left.\operatorname{Stab}_{H}\left(\mathcal{B}_{2}\right)\right|_{B}, B \in \mathcal{B}_{2}$, must be cyclic of order $p^{2}$ as a Sylow $p$-subgroup of $\left.\operatorname{fix}_{P}\left(\mathcal{B}_{2}\right)\right|_{B}=\left.\left\langle\tau_{2}\right\rangle\right|_{B}, B \in \mathcal{B}_{2}$, and the fact that $\operatorname{Stab}_{P}(B)=\operatorname{fix}_{P}\left(\mathcal{B}_{2}\right)$ as $P / \mathcal{B}_{2}$ has order $p$ and so is regular. As the blocks of $\mathcal{D}$ contained within a block $B \in \mathcal{B}_{2}$ form a complete block system of $\left.\operatorname{Stab}_{H}\left(\mathcal{B}_{2}\right)\right|_{B},\left.\operatorname{Stab}_{H}\left(\mathcal{B}_{2}\right)\right|_{B}$ is imprimitive. By Theorem 7 we have that $\left.\operatorname{Stab}_{H}\left(\mathcal{B}_{2}\right)\right|_{B}$ has a unique Sylow $p$-subgroup, which is $\left.\left\langle\tau_{2}\right\rangle\right|_{B}$, and

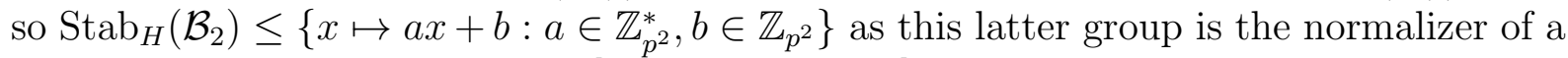
regular cyclic subgroup in $S_{p^{2}}$ by [6, Corollary 4.2B]. Additionally, as a Sylow $p$-subgroup of $\left.\operatorname{Stab}_{H}\left(\mathcal{B}_{2}\right)\right|_{B}$ has order $p^{2}$, if $x \mapsto a x+b$ is contained in $\left.\operatorname{Stab}_{H}\left(\mathcal{B}_{2}\right)\right|_{B}$, then $\operatorname{gcd}(|a|, p)=1$. By the Embedding Theorem [21, Theorem 1.2.6], we have that $h(i, j)=\left(\omega(i), \alpha_{i} j+c_{i}\right)$, $\omega \in S_{p}, \alpha_{i} \in \mathbb{Z}_{p^{2}}^{*}$ of order relatively prime to $p$, and $c_{i} \in \mathbb{Z}_{p^{2}}$. As $\operatorname{fix}_{P}(\mathcal{D})$ has order $p^{p}$ and $|P|=p^{p+2}$, we have that a Sylow $p$-subgroup of $H / \mathcal{D}$ has order $p^{2}$ and, as $\mathcal{B}_{2}$ is a complete block system of $H, H / \mathcal{D}$ is imprimitive. By [15, Theorem 4 and Lemma 1], we have that $H / \mathcal{D}$ is conjugate to a subgroup of $S_{p} \times S_{p}$.

Assume for the moment that $H / \mathcal{D} \leq S_{p} \times S_{p}$. Then $\alpha_{i} \equiv \alpha_{i^{\prime}}(\bmod p)$ and $c_{i} \equiv$ $c_{i^{\prime}}(\bmod p)$ for every $i, i^{\prime} \in \mathbb{Z}_{p}$. We may thus assume without loss of generality that $c_{i} \equiv 0(\bmod p)$ for every $i \in \mathbb{Z}_{p}$. As $\left.\tau_{2}^{p}\right|_{B} \in H$ for all $B \in \mathcal{B}_{2}$, we may assume without loss of generality that $c_{i}=0$ for all $i \in \mathbb{Z}_{p}$. Equivalently, $c_{i}=a+p b_{i}$ for some $a, b_{i} \in \mathbb{Z}_{p}$.

If $\alpha_{k}=c+b p$ for some $b, c \in \mathbb{Z}_{p}$ with $b \neq 0$, then $\alpha_{k}^{p} \equiv c^{p}\left(\bmod p^{2}\right)$, and so $p$ divides $\left|\alpha_{k}\right|$, which is not possible. Hence $\alpha_{k}=c$ for all $k \in \mathbb{Z}_{p}$. The result will then follow provided there exists $\beta \in \operatorname{Aut}\left(\mathbb{Z}_{p} \times \mathbb{Z}_{p^{2}}\right)$ such that $\beta H \beta^{-1} / \mathcal{D} \leq S_{p} \times S_{p}$.

Now, as $H / \mathcal{D}$ is conjugate in $S_{p^{2}}$ to a subgroup of $S_{p} \times S_{p}$, there exists $\delta \in S_{\mathbb{Z}_{p} \times \mathbb{Z}_{p^{2}}}$ such that $\delta H \delta^{-1} \leq\left\{(i, j) \mapsto\left(\omega(i), \alpha j+a+p b_{i}: \omega \in S_{p}, a, b_{i} \in \mathbb{Z}_{p}\right\}=L\right.$. By $[8$, Lemma 8] (we remark that the statement of [8, Lemma 8] holds for a Cayley graph $\Gamma$, but the proof only really depends on a Sylow $p$-subgroup of Aut $(\Gamma)$ being $P$ ) there exists $\gamma \in L$ such that $\gamma \delta\left\langle\tau_{1}, \tau_{2}\right\rangle \delta^{-1} \gamma^{-1}=\left\langle\tau_{1}, \tau_{2}\right\rangle$, and, of course, $\gamma \delta H \delta^{-1} \gamma^{-1} \leq L$. As $\gamma \delta\left\langle\tau_{1}, \tau_{2}\right\rangle \delta^{-1} \gamma^{-1}=\left\langle\tau_{1}, \tau_{2}\right\rangle$, by [6, Corollary 4.2B], $\gamma \delta \in \operatorname{Aut}\left(\mathbb{Z}_{p} \times \mathbb{Z}_{p^{2}}\right) \cdot\left\langle\tau_{1}, \tau_{2}\right\rangle$, so we may assume that $\gamma \delta=\beta \in \operatorname{Aut}\left(\mathbb{Z}_{p} \times \mathbb{Z}_{p^{2}}\right)$. Then $\beta H \beta^{-1} / \mathcal{D} \leq S_{p} \times S_{p}$ and the result follows.

Lemma 21 Let $H \leq S_{\mathbb{Z}_{p} \times \mathbb{Z}_{p^{2}}}$ have Sylow p-subgroup $P=\left\langle\tau_{1}, \tau_{2},\left.\tau_{2}^{p}\right|_{B}: B \in \mathcal{B}_{2}\right\rangle$. Also suppose that $H \leq\left\{(i, j) \mapsto\left(\omega(i), \alpha j+a+p b_{i}\right): \omega \in \operatorname{AGL}(1, p), \alpha \in \mathbb{Z}_{p^{2}}^{*}, a, b_{i} \in \mathbb{Z}_{p}\right\}$. Then $H=A \cdot P$ for some $A \leq \operatorname{Aut}\left(\mathbb{Z}_{p} \times \mathbb{Z}_{p^{2}}\right)$. 
Proof. Straightforward computations will show that if $h \in H$ then $h$ normalizes $\left\langle\tau_{2}\right\rangle$, and as each $\omega \in \operatorname{AGL}(1, p)$, we see that $h^{-1} \tau_{1} h \in\left\langle\tau_{1},\left.\tau_{2}^{p}\right|_{B}: B \in \mathcal{B}\right\rangle$. We conclude that $H$ normalizes $P$. By $[8$, Lemma 8$]$, we have that any two transitive subgroups of $P$ isomorphic to $\mathbb{Z}_{p} \times \mathbb{Z}_{p^{2}}$ are conjugate in $P$. The result follows then follows from Lemma 16.

This completes our consideration of overgroups of $p$-groups given in Theorem 12 (iv), and we now begin to consider the overgroups of those $p$-groups given in Theorem $12(\mathrm{v})$.

Lemma 22 Let $H \leq S_{\mathbb{Z}_{p} \times \mathbb{Z}_{p^{2}}}$ be transitive with Sylow p-subgroup $P=\left\langle\tau_{1}, \tau_{2},\left.\tau_{1}\right|_{B}: B \in\right.$ $\left.\mathcal{B}_{1,1}\right\rangle$. Assume that $H$ admits a complete block system $\mathcal{D}$ with blocks of prime size and $H / \mathcal{D}$ is imprimitive. Then $H$ admits a complete block system with blocks formed by the orbits of $\left\langle\tau_{1}\right\rangle$.

Proof. Suppose that $\mathcal{D}$ is formed by the orbits of $K \neq\left\langle\tau_{1}\right\rangle$, where $K \leq\left\langle\tau_{1}, \tau_{2}\right\rangle$. Then a Sylow $p$-subgroup of $H / \mathcal{D}$ is isomorphic to $\mathbb{Z}_{p} \prec \mathbb{Z}_{p}$, and so admits a unique complete block system $\mathcal{F}$ of $p$ blocks of size $p$. As a Sylow $p$-subgroup of $H$ is $P, \mathcal{F}$ must be formed by the orbits of $\left\langle\tau_{1}\right\rangle / \mathcal{D}$. Then $H$ admits a complete block system $\mathcal{E}$ consisting of $p$ blocks of size $p^{2}$ induced by $\mathcal{F}$, so that $\mathcal{E}$ is formed by the orbits of $\left\langle\tau_{1}, \tau_{2}^{p}\right\rangle$. Hence $\mathcal{E}=\mathcal{B}_{1,1}$. Then $\left.\tau_{1}\right|_{B} \in \operatorname{fix}_{H}\left(\mathcal{B}_{1,1}\right)$ for every $B \in \mathcal{B}_{1,1}$. Let $B \in \mathcal{B}_{1,1}$, and $L=\left\langle\left.\tau_{1}\right|_{B}\right\rangle^{\operatorname{Stab}_{H}(B)}$. Note that every element of order $p$ of $L$ fixes all points outside $B$. The only elements of $P$ with this property are elements of $\left\langle\left.\tau_{1}\right|_{B}: B \in \mathcal{B}_{1,1}\right\rangle$, as $P$ admits a complete block system $\mathcal{L}$ formed by the orbits of $\left\langle\tau_{1}\right\rangle$ and $P / \mathcal{L} \cong \mathbb{Z}_{p^{2}}$. If $\left.L\right|_{B}$ is transitive, then $\left.L\right|_{B}$ contains a transitive Sylow $p$-subgroup, say $P_{B}$. We conclude that $1_{S_{p}} \prec P_{B} \leq H$, and so a Sylow $p$-subgroup of $H$ has order at least $p \cdot\left(p^{2}\right)^{p}$. However, $P$ has order $p^{2} \cdot p^{p}$ and so $p=1$, a contradiction. Thus $\left.L\right|_{B}$ is intransitive, and so the orbits of $\left.L\right|_{B}$ form a complete block system of $\left.\operatorname{Stab}_{H}(B)\right|_{B}$, which is necessarily formed by the orbits of $\left\langle\left.\tau_{1}\right|_{B}\right\rangle$. By [6, Exercise 1.5.10], the set of all blocks conjugate to an orbit of $\left\langle\left.\tau_{1}\right|_{B}\right\rangle$ forms a complete block system of $H$, and so $H$ admits a complete block system formed by the orbits of $\left\langle\tau_{1}\right\rangle$.

Lemma 23 Let $p \geq 5$ and $H \leq S_{\mathbb{Z}_{p} \times \mathbb{Z}_{p^{2}}}$ be transitive with Sylow p-subgroup $P=$ $\left\langle\tau_{1}, \tau_{2},\left.\tau_{1}\right|_{B}: B \in \mathcal{B}_{1,1}\right\rangle$. Assume that $H$ admits a complete block system $\mathcal{D}$ with blocks of prime size and $H / \mathcal{D}$ is primitive. Then $\mathcal{D}$ is formed by the orbits of $\left\langle\tau_{1}\right\rangle$.

Proof. Assume $\mathcal{D}$ is not formed by the orbits of $\left\langle\tau_{1}\right\rangle$, so that a Sylow $p$-subgroup of $H / \mathcal{D}$ is $\mathbb{Z}_{p} \geq \mathbb{Z}_{p}$. As $H / \mathcal{D}$ is primitive and $\mathbb{Z}_{p} \geq \mathbb{Z}_{p}$ contains a regular subgroup isomorphic to $\mathbb{Z}_{p^{2}}$, we have that $H / \mathcal{D}$ is doubly-transitive as $\mathbb{Z}_{p^{2}}$ is a Burnside group [6, Theorem 3.5A]. Perusing the list of doubly-transitive groups of degree $p^{2}[19$, Theorem 1.1] (or [16, Theorem 3]) that contain a regular cyclic subgroup and observing that no doubly-transitive group of primesquared degree contained in any $\operatorname{P\Gamma L}(n, k)$ contains a Sylow $p$-subgroup isomorphic to $\mathbb{Z}_{p}\left\langle\mathbb{Z}_{p}\right.$ by Lemma 8 , we have that $A_{p^{2}} \leq H / \mathcal{D}$. Thus $A_{p}\left\langle A_{p} \leq H / \mathcal{D}\right.$ and $A_{p}$ is nonsolvable as $p \geq 5$. Let $L \leq H$ be maximal such that $L / \mathcal{D}=A_{p} 2 A_{p}$. Then $L / \mathcal{D}$ is imprimitive, and so by Lemma $22, L$ admits a complete block system $\mathcal{I}$ formed by the orbits of $\left\langle\tau_{1}\right\rangle$. Then a Sylow $p$-subgroup of $L / \mathcal{I}$ is cyclic of order $p^{2}$ as $P / \mathcal{I}$ is cyclic. As $L / \mathcal{D}=A_{p} 2 A_{p}$, 
$L / \mathcal{D}$ admits a complete block system $\mathcal{E}$ necessarily formed by the orbits of $\left\langle\tau_{1}\right\rangle / \mathcal{D}$, and so $L$ admits a complete block system $\mathcal{F}$ consisting of $p$ blocks of size $p^{2}$ formed by the orbits of $\left\langle\tau_{1}, \tau_{2}^{p}\right\rangle$. Note that $\mathcal{I} \prec \mathcal{F}$. As $A_{p}=(L / \mathcal{D}) / \mathcal{E}=L / \mathcal{F}$, we have that $L / \mathcal{I}$ is nonsolvable as $p \geq 5$. As a Sylow $p$-subgroup of $L / \mathcal{I}$, is cyclic and imprimitive, by Theorem 7 we have that $L / \mathcal{I}$ contains a normal transitive cyclic subgroup, a contradiction as the normalizer of a $p^{2}$-cycle in $S_{p^{2}}$ is isomorphic to $\left\{x \mapsto a x+b: a \in \mathbb{Z}_{p^{2}}^{*}, b \in \mathbb{Z}_{p^{2}}\right\}$ by $[6$, Corollary 4.2B].

Definition 24 View each element of $\mathbb{Z}_{p} \times \mathbb{Z}_{p^{2}}$ uniquely as $(i, j+k p)$, where $i, j, k \in \mathbb{Z}_{p}$. Define $\alpha_{2}: \mathbb{Z}_{p} \times \mathbb{Z}_{p^{2}}$ by $\alpha_{2}(i, j+k p)=(i+k, j+k p)$. Note that $\alpha_{2}^{-1}(i, j+k p)=(i-k, j+k p)$. Straightforward computations will then show that $\alpha_{2}^{-1} \tau_{2} \alpha_{2}(i, j+k p)=(i, j+1+k p)$ if $j \neq p-1$ while $\alpha_{2}^{-1} \tau_{2} \alpha_{2}(i, p-1+k p)=(i-1,(k+1) p)$. Then $\mathcal{B}_{1,1}=\left\{B_{j}: j \in \mathbb{Z}_{p}\right\}$, where $B_{j}=\left\{(i, j+k p): i, k \in \mathbb{Z}_{p}\right\}$. We then have that $\alpha_{2}^{-1} \tau_{2} \alpha_{2}=\tau_{2}\left(\left.\tau_{1}^{-1}\right|_{B_{p-1}}\right)$. It is not hard to see that $\alpha_{2}$ commutes with $\left.\tau_{1}\right|_{B}$ for every $B \in \mathcal{B}_{1,1}$ and $\alpha_{2}^{-1} \tau_{2}^{p} \alpha_{2}=\tau_{1}^{-1} \tau_{2}^{p}$. Then $\alpha_{2}$ normalizes $\left\langle\left.\tau_{1}\right|_{B}, \tau_{2}: B \in \mathcal{B}_{1,1}\right\rangle$. We now view $\mathbb{Z}_{p} \times \mathbb{Z}_{p^{2}}$ in the usual fashion. For $a \in \mathbb{Z}_{p^{*}}$, define $\bar{a}: \mathbb{Z}_{p} \times \mathbb{Z}_{p^{2}} \mapsto \mathbb{Z}_{p} \times \mathbb{Z}_{p^{2}}$ by $\bar{a}(i, j)=\left(a^{-1} i, j\right)$. Note that $\bar{a}^{-1} \tau_{1} \bar{a}=\tau_{1}^{a}$ and $\bar{a}$ commutes with $\tau_{2}$.

Lemma 25 Let $H \leq S_{\mathbb{Z}_{p} \times \mathbb{Z}_{p^{2}}}$ be transitive with Sylow p-subgroup $P=\left\langle\tau_{1}, \tau_{2},\left.\tau_{1}\right|_{B}\right.$ : $\left.B \in \mathcal{B}_{1,1}\right\rangle$. If $p \geq 5$, then for $c=0$ or 1 , and $a \in \mathbb{Z}_{p}^{*}, \alpha_{2}^{-c} \bar{a}^{-1} H \bar{a} \alpha_{2}^{c} \leq\{(i, j) \mapsto$ $\left.\left(\beta_{j(\bmod p)}(i), \alpha j+b\right): \beta_{j(\bmod p)} \in S_{p}, \alpha \in \mathbb{Z}_{p^{2}}^{*}, b \in \mathbb{Z}_{p^{2}}\right\}$.

Proof. By Lemma 18, we have that $H$ is imprimitive and a minimal complete block system $\mathcal{D}$ of $H$ has blocks of size $p$ formed by the orbits of some subgroup $K \leq G$. As $H / \mathcal{D}$ is imprimitive or primitive, by Lemma 22 or 23 , respectively, we have that $H$ admits a complete block system formed by the orbits of $\left\langle\tau_{1}\right\rangle$. We thus assume without loss of generality that $\mathcal{D}$ is formed by the orbits of $\left\langle\tau_{1}\right\rangle$.

As $\mathcal{D}$ is formed by the orbits of $\left\langle\tau_{1}\right\rangle$, and a Sylow $p$-subgroup of $\operatorname{fix}_{H}(\mathcal{D})$ is $\left\langle\left.\tau_{1}\right|_{B}\right.$ : $\left.B \in \mathcal{B}_{1,1}\right\rangle$, by Lemma $10 \mathcal{B}_{1,1}$ is a complete block system of $H$. Thus $H / \mathcal{D}$ is imprimitive. Also, a Sylow $p$-subgroup of $H / \mathcal{D}$ is regular and cyclic as a Sylow $p$-subgroup of $P / \mathcal{D}$ is regular and cyclic. By Theorem 7 we conclude that $H / \mathcal{D}$ contains a normal regular cyclic subgroup, and so if $h \in H$, then $h(i, j)=\left(\beta_{j}(i), \alpha j+b\right), \beta_{j} \in S_{p}, \alpha \in \mathbb{Z}_{p^{2}}^{*}$ of order not divisible by $p$, and $b \in \mathbb{Z}_{p^{2}}$.

As $\mathcal{B}_{1,1}$ is a complete block system of $H$ and as $P$ is a Sylow $p$-subgroup of $H$, we have that a Sylow $p$-subgroup of $\left.\operatorname{Stab}_{H}(B)\right|_{B}$ is elementary abelian and $\left.\operatorname{Stab}_{H}(B)\right|_{B}$ is imprimitive, $B \in \mathcal{B}_{1,1}$. By [15, Theorem 4], we have that $\left.\operatorname{Stab}_{H}(B)\right|_{B}$ is permutation isomorphic to a subgroup of $S_{p} \times S_{p}$, and so $\left.\operatorname{Stab}_{H}(B)\right|_{B}$ admits a complete block system $\mathcal{E}$ consisting of $p$ blocks of size $p$ and no block of $\mathcal{E}$ is contained in $\mathcal{D}$. By [6, Exercise 1.5.10], $H$ admits a complete block system $\mathcal{F}$ whose blocks consist of those blocks conjugate in $H$ to a block of $\mathcal{E}$. Then $\mathcal{F}$ is genuine, being formed by the orbits of $\left\langle\tau_{1}^{c} \tau_{2}^{a p}\right\rangle, c=0,1$, $a \in \mathbb{Z}_{p}^{*}$, and if $c=0$, then we may and do take $a=1$. If $c=1$, then $\alpha_{2}^{-c} \bar{a}^{-1} \tau_{1} \bar{a} \alpha_{2}^{c}=\tau_{1}^{a}$ and $\alpha_{2}^{-c} \tau_{2}^{a p} \alpha_{2}^{c}=\tau_{1}^{-a} \tau_{2}^{a p}$. Thus $\alpha_{2}^{-1} \bar{a}^{-1} \tau_{1} \tau_{2}^{a p} \bar{a} \alpha_{2}=\tau_{2}^{a p}$. Also, $\alpha_{2}$ normalizes $P$ as does $\bar{a}$. 
We may then assume without loss of generality that $\mathcal{F}$ is formed by the orbits of $\left\langle\tau_{2}^{p}\right\rangle$ by replacing $H$ with $\alpha_{2}^{-c} \bar{a}^{-1} H \bar{a} \alpha_{2}^{c}$ for $c=0,1$. Then $\left.\operatorname{Stab}_{H}(B)\right|_{B} \leq S_{p} \times S_{p}$.

Now let $h \in H$. Then $h(i, j)=\left(\beta_{j}(i), \alpha j+b\right)$, where $\beta_{j} \in S_{p}, \alpha \in \mathbb{Z}_{p^{2}}^{*}$ has order relatively prime to $p$, and $b \in \mathbb{Z}_{p^{2}}$. Also, $h^{-1}\left\langle\tau_{1}, \tau_{2}\right\rangle h \leq H$ and is a regular subgroup isomorphic to $\mathbb{Z}_{p} \times \mathbb{Z}_{p^{2}}$. Setting $K=\left(\left\langle\tau_{1}, \tau_{2}, h^{-1}\left\langle\tau_{1}, \tau_{2}\right\rangle h\right\rangle\right)^{(2)}$, we see that a Sylow $p$-subgroup of $K$ is either $\left\langle\tau_{1}, \tau_{2}\right\rangle$ or $P$ by Theorem 12. In the latter case, by [8, Lemma 9], and in the former case by a Sylow Theorem, there exists $k \in K$ such that $k^{-1} h^{-1}\left\langle\tau_{1}, \tau_{2}\right\rangle h k=\left\langle\tau_{1}, \tau_{2}\right\rangle$. Then $k h$ normalizes $\left\langle\tau_{1}, \tau_{2}\right\rangle$. Observe now that $\left\langle\tau_{1}, \tau_{2}, h^{-1}\left\langle\tau_{1}, \tau_{2}\right\rangle h\right\rangle \leq \mathbb{Z}_{p^{2}} S_{p}$ as $H / \mathcal{D}$ has a normal cyclic Sylow $p$-subgroup of order $p^{2}$, in which case $K \leq \mathbb{Z}_{p^{2}} \imath S_{p}$ as $\mathbb{Z}_{p^{2}} 2 S_{p}$ is 2 -closed. Thus $K / \mathcal{D}=\left\langle\tau_{2}\right\rangle / \mathcal{D}$, and so by replacing $k$ with $\tau_{2}^{d} k$ for appropriate $d \in \mathbb{Z}$, we may assume without loss of generality that $k \in \operatorname{fix}_{K}(\mathcal{D})$. Let $B^{\prime} \in \mathcal{B}_{1,1}$. Then there exists $e_{B^{\prime}} \in \mathbb{Z}$ such that $k h \tau_{2}^{e_{B^{\prime}}}\left(B^{\prime}\right)=B^{\prime}$, and there exists $d_{B^{\prime}} \in \mathbb{Z}_{p}$ such that $\tau_{2}^{-d_{B^{\prime}}}\left(k h \tau_{2}^{e_{B^{\prime}}}\right) \tau_{2}^{d_{B^{\prime}}}$ stabilizes $B$. Then $\left.\tau_{2}^{-d_{B^{\prime}}}\left(k h \tau_{2}^{e_{B^{\prime}}}\right) \tau_{2}^{d_{B^{\prime}}}\right|_{B} \leq S_{p} \times S_{p}$. As $\left.\tau_{2}^{-d_{B^{\prime}}} k \tau_{2}^{d_{B^{\prime}}}\right|_{B} \leq S_{p} \times S_{p}$ as $\tau_{2}^{-d_{B^{\prime}}} k \tau_{2}^{d_{B^{\prime}}} \in \operatorname{Stab}_{H}(B)$, we see that $\left.\tau_{2}^{-d_{B^{\prime}}} h \tau_{2}^{e_{B}^{\prime}} \tau_{2}^{d_{B^{\prime}}}\right|_{B} \leq S_{p} \times S_{p}$ for every $B \in \mathcal{B}_{1,1}$. As $\tau_{2}(i, j)=(i, j+1)$, we conclude that $\left.h \tau_{2}^{e_{B}}\right|_{B^{\prime}} \leq S_{p} \times S_{p}$. Hence if $i \equiv i^{\prime}(\bmod p)$, then $\beta_{i}=\beta_{i^{\prime}}$, and the result follows.

Lemma 26 Let $H \leq S_{\mathbb{Z}_{p} \times \mathbb{Z}_{p^{2}}}$ be transitive and 2-closed with Sylow p-subgroup $P=$ $\left\langle\tau_{1}, \tau_{2},\left.\tau_{1}\right|_{B}: B \in \mathcal{B}_{1,1}\right\rangle$. If $H \leq\left\{(i, j) \mapsto\left(\beta_{j}(\bmod p)(i), \alpha j+b\right): \beta_{j}(\bmod p) \in \operatorname{AGL}(1, p)\right.$, $\left.\alpha \in \mathbb{Z}_{p^{2}}^{*}, b \in \mathbb{Z}_{p^{2}}\right\}$, then there exists $D \leq \mathbb{Z}_{p}^{*}$ and $A \leq \operatorname{Aut}\left(\mathbb{Z}_{p} \times \mathbb{Z}_{p^{2}}\right)$ such that $H=$ $A \cdot\left\{(i, j) \mapsto\left(d_{j}(\bmod p) i+c_{j}(\bmod p), j+b\right): d_{j(\bmod p)} \in D, c_{j}(\bmod p) \in \mathbb{Z}_{p}\right.$, and $\left.b \in \mathbb{Z}_{p^{2}}\right\}$.

ProOf. First observe that $H$ admits a complete block system $\mathcal{B}$ of $p^{2}$ blocks of size $p$ formed by the orbits of $\left\langle\tau_{1}\right\rangle$. As $H \leq\left\{(i, j) \mapsto\left(\beta_{j}(\bmod p)(i), \alpha j+b\right): \beta_{j}(\bmod p) \in\right.$ $\left.\operatorname{AGL}(1, p), \alpha \in \mathbb{Z}_{p^{2}}^{*}, b \in \mathbb{Z}_{p^{2}}\right\}$ we have that $\left.\operatorname{fix}_{H}(\mathcal{B})\right|_{B} \leq \operatorname{AGL}(1, p)$. Let $\left.\operatorname{fix}_{H}(\mathcal{B})\right|_{B}=$ $D \cdot\left(\mathbb{Z}_{p}\right)_{L}$, where $D \leq \mathbb{Z}_{p}^{*}$. We observe that such a $D$ exists as fix $\left.{ }_{H}(\mathcal{B})\right|_{B}$ has a unique Sylow $p$-subgroup $\left(\mathbb{Z}_{p}\right)_{L}$, and [6, Corollary 4.2B]. Define an equivalence relation $\equiv$ on the blocks of $\mathcal{B}$ by $B \equiv B^{\prime}$ if and only if whenever $h \in \operatorname{fix}_{H}(\mathcal{B})$ then $\left.h\right|_{B}$ is a $p$-cycle if and only if $\left.h\right|_{B^{\prime}}$ is also a $p$-cycle. Clearly the equivalence classes of $\equiv$ form $\mathcal{B}_{1,1}$ as $\left\langle\left.\tau_{1}\right|_{B_{1,1}}: B_{1,1} \in \mathcal{B}_{1,1}\right\rangle$ is a Sylow $p$-subgroup of $\operatorname{fix}_{H}(\mathcal{B})$. By Lemma 10 we have that if $h \in \operatorname{fix}_{H}(\mathcal{B})$, then $\left.h\right|_{B_{1,1}} \in \operatorname{fix}_{H}(\mathcal{B})$ for every $B_{1,1} \in \mathcal{B}_{1,1}$, where as usual $\left.h\right|_{B_{1,1}}$ is the permutation equal to $h$ on $B_{1,1}$ and the identity on every other block of $\mathcal{B}_{1,1}$. Thus $\operatorname{fix}_{H}(\mathcal{B})=\{(i, j) \mapsto$ $\left.\left(d_{j(\bmod p)} i+c_{j(\bmod p)}, j\right): d_{j(\bmod p)} \in D, c_{j}(\bmod p) \in \mathbb{Z}_{p}\right\}$. Furthermore, as $\left\langle\tau_{2}\right\rangle / \mathcal{B} \triangleleft H / \mathcal{B}$ and $\operatorname{fix}_{H}(\mathcal{B}) \triangleleft H$, we have that $K=\left\{(i, j) \mapsto\left(d_{j}(\bmod p) i+c_{j}(\bmod p), j+b\right): d_{j}(\bmod p) \in\right.$ $D, c_{j}(\bmod p) \in \mathbb{Z}_{p}$, and $\left.b \in \mathbb{Z}_{p^{2}}\right\} \triangleleft H$.

Now let $h \in H$. Then $h^{-1}\left\langle\tau_{1}, \tau_{2}\right\rangle h \leq K$ and is contained in a Sylow $p$-subgroup of $K$. Hence there exists $k_{1} \in K$ such that $k_{1}^{-1} h^{-1}\left\langle\tau_{1}, \tau_{2}\right\rangle h k_{1} \leq P$. By [8, Lemma 9], there exists $k_{2} \in P$ such that $k_{2}^{-1} k_{1}^{-1} h^{-1}\left\langle\tau_{1}, \tau_{2}\right\rangle h k_{1} k_{2}=\left\langle\tau_{1}, \tau_{2}\right\rangle$. The result then follows by Lemma 16 .

This completes our consideration of overgroups of $p$-groups given in Theorem $12(\mathrm{v})$, and we now begin to consider the overgroups of those $p$-groups given in Theorem 12 (vi). 
Lemma 27 Let $\gamma: \mathbb{Z}_{p} \times \mathbb{Z}_{p^{2}} \mapsto \mathbb{Z}_{p} \times \mathbb{Z}_{p^{2}}$ by $\gamma(i, j)=(i+[a j(\bmod p)], j+i b p), a, b \in \mathbb{Z}_{p}^{*}$, and $P=\left\langle\tau_{1}, \tau_{2}, \gamma\right\rangle$ be of order $p^{4}$. Then the only nontrivial complete block systems of $P$ are formed by the orbits of $\left\langle\tau_{2}^{p}\right\rangle$ and $\left\langle\tau_{1}, \tau_{2}^{p}\right\rangle$.

Proof. Straightforward computations will show that $\gamma^{-1}(i, j)=(i-[a j(\bmod p)], j-$ $[i-(a j(\bmod p))] b p)$. Then $\gamma^{-1} \tau_{1} \gamma=\tau_{1} \tau_{2}^{-b p}$, while $\gamma^{-1} \tau_{2} \gamma=\tau_{1}^{-a} \tau_{2}^{1+a b p}$. Then $P$ admits a complete block system $\mathcal{B}$ formed by the orbits of $\left\langle\tau_{2}^{p}\right\rangle$ as $\mathrm{Z}(P)=\left\langle\tau_{2}^{p}\right\rangle$ where $\mathrm{Z}(P)$ is the center of $P$. Also, $K=\left\langle\tau_{1}, \tau_{2}^{p}, \gamma\right\rangle \triangleleft P$ as it is a subgroup of index $p$ (or just using direct computation) in $P$, and so $P$ admits $\mathcal{B}_{1,1}$ as a complete block system. Note that any subgroup $L$ of $P$ of order $p^{3}$ different from $\left\langle\tau_{1}, \tau_{2}\right\rangle$ must contain a subgroup of $\left\langle\tau_{1}, \tau_{2}\right\rangle$ of order $p^{2}$, so $L$ contains either $\left\langle\tau_{1}, \tau_{2}^{p}\right\rangle$ or $\left\langle\tau_{1}^{c} \tau_{2}\right\rangle$. In the latter case, as $L \triangleleft P$ as $L$ is of index $p$ in $P, \gamma^{-1} \tau_{1}^{c} \tau_{2} \gamma=\tau_{1}^{c-a} \tau_{2}^{1+b p(a-c)}$ is contained in $L$. If $c \neq a$, then $L \geq\left\langle\tau_{1}^{c} \tau_{2}, \tau_{1}^{c-a} \tau_{2}^{1+b p(a-c)}\right\rangle=\left\langle\tau_{1}, \tau_{2}\right\rangle$, a contradiction. If $c=a$, then $L$ contains $\tau_{2}$ as well, and $L$ contains all of $\left\langle\tau_{1}, \tau_{2}\right\rangle$, again a contradiction. Thus $L$ contains $\left\langle\tau_{1}, \tau_{2}^{p}\right\rangle$. As if $\mathcal{D}$ is a complete block system of $P$ with blocks of size $p^{2}$, then $P / \mathcal{D}$ has order $p$, we must have that $\mathcal{D}$ is formed by the orbits of a subgroup of $P$ of order $p^{3}$ that is intransitive, and contains $\left\langle\tau_{1}, \tau_{2}^{p}\right\rangle$, and so must be $\mathcal{B}_{1,1}$. By Lemma 15 , any complete block system $\mathcal{D}$ of $P$ with blocks of prime size is formed by the orbits of $\left\langle\tau_{2}^{p}\right\rangle$ or $\left\langle\tau_{1} \tau_{2}^{c p}\right\rangle$ for some $c \in \mathbb{Z}_{p}$. If the latter case occurs, then note that the orbits of $\gamma$ are not contained in the orbits of $\left\langle\tau_{1} \tau_{2}^{c p}\right\rangle$ for any $c \in \mathbb{Z}_{p}$ (the orbit of $\langle\gamma\rangle$ that contains $(1,1)$ also contains $(1+a, 1+b p)$ and $(1+2 a, 1+2 b p+a b p)$, for example), and so $\operatorname{fix}_{P}(\mathcal{D})$ has order $p$. Thus if $\mathcal{D}$ is formed by the orbits of $\left\langle\tau_{1} \tau_{2}^{c p}\right\rangle$, then $\left\langle\tau_{1} \tau_{2}^{c p}\right\rangle \triangleleft P$. However, $\gamma^{-1} \tau_{1} \tau_{2}^{c p} \gamma=\tau_{1} \tau_{2}^{c p-b p}$. Thus $\mathcal{D}$ is not formed by the orbits of $\left\langle\tau_{1} \tau_{2}^{c p}\right\rangle$ and so $\mathcal{D}$ is formed by $\left\langle\tau_{2}^{p}\right\rangle$. The result then follows.

Lemma 28 Let $\gamma: \mathbb{Z}_{p} \times \mathbb{Z}_{p^{2}} \mapsto \mathbb{Z}_{p} \times \mathbb{Z}_{p^{2}}$ by $\gamma(i, j)=(i+[a j(\bmod p)], j+i b p), a, b \in \mathbb{Z}_{p}^{*}$, and $P=\left\langle\tau_{1}, \tau_{2}, \gamma\right\rangle$ be of order $p^{4}$. Let $H \leq S_{\mathbb{Z}_{p} \times \mathbb{Z}_{p^{2}}}$ have Sylow p-subgroup $P$ and admit $\mathcal{B}_{1,1}$ as a complete block system. Then there exists $K \leq H$ with Sylow $p$-subgroup $P$ such that $K / \mathcal{B}_{1,1}=H / \mathcal{B}_{1,1}$ and $K$ also admits a complete block system formed by the orbits of $\left\langle\tau_{2}^{p}\right\rangle$.

Proof. Let $L=\left\langle\tau_{1}, \tau_{2}^{p}, \gamma\right\rangle$, so that $L$ is a Sylow $p$-subgroup of $\operatorname{fix}_{H}\left(\mathcal{B}_{1,1}\right)$. Let $h \in H$, so that $h^{-1} L h \leq \operatorname{fix}_{H}\left(\mathcal{B}_{1,1}\right)$ is also a Sylow $p$-subgroup of $\operatorname{fix}_{H}\left(\mathcal{B}_{1,1}\right)$. Hence there exists $\beta_{h} \in$ $\operatorname{fix}_{H}\left(\mathcal{B}_{1,1}\right)$ such that $\beta_{h}^{-1} h^{-1} L h \beta_{h}=L$, so that $h \beta_{h}$ normalizes $L$ and $h \beta_{h} / \mathcal{B}_{1,1}=h / \mathcal{B}_{1,1}$. Let $K=\left\langle h \beta_{h}: h \in H\right\rangle$, so that $K / \mathcal{B}_{1,1}=H / \mathcal{B}_{1,1}$ and $L \triangleleft K$. Note that the center Z $(L)$ of $L$ is $\left\langle\tau_{2}^{p}\right\rangle$ and, as the center of a group is characteristic, $\left\langle\tau_{2}^{p}\right\rangle \triangleleft K$ so that $K$ admits the required complete block system.

Lemma 29 Let $p \geq 3, \gamma: \mathbb{Z}_{p} \times \mathbb{Z}_{p^{2}} \mapsto \mathbb{Z}_{p} \times \mathbb{Z}_{p^{2}}$ by $\gamma(i, j)=(i+[a j(\bmod p)], j+i b p)$, $a, b \in \mathbb{Z}_{p}^{*}$, and $P=\left\langle\tau_{1}, \tau_{2}, \gamma\right\rangle$ be of order $p^{4}$. If $H \leq S_{\mathbb{Z}_{p} \times \mathbb{Z}_{p^{2}}}$ with Sylow p-subgroup $P$, then $H$ admits a complete block system formed by the orbits of $\left\langle\tau_{2}^{p}\right\rangle$ or $\left\langle\tau_{1}, \tau_{2}\right\rangle \triangleleft H$.

Proof. Examining the list of primitive groups that contain a regular abelian subgroup given by [19, Theorem 1.1], we see that $H$ is not primitive. As any complete block system 
of $H$ is also a complete block system of $P$, by Lemma 27 we have that either the result follows or $\mathcal{B}_{1,1}$ is the only nontrivial complete block system of $H$. By [6, Exercise 1.5.10] we have that $\left.\operatorname{Stab}_{H}(B)\right|_{B}$ is primitive for every $B \in \mathcal{B}_{1,1}$. Additionally, $\left.\operatorname{Stab}_{H}(B)\right|_{B}$ contains a Sylow $p$-subgroup of order $p^{3}$ with a normal elementary abelian subgroup of order $p^{2}$ (and does not contain a regular cyclic subgroup as $p \geq 3$ - see [15, Lemma 4]). By [19, Theorem 1.1], we have that $\left.\operatorname{Stab}_{H}(B)\right|_{B} \leq \operatorname{AGL}(2, p)$ for every $B \in \mathcal{B}_{1,1}$. By the Embedding Theorem [21, Theorem 1.2.6], we have that $H$ is permutation isomorphic to a subgroup of $H / \mathcal{B}_{1,1}$ 々 $\operatorname{AGL}(2, p)$.

By Lemma 28, there exists $P \leq K \leq H$ such that $K / \mathcal{B}_{1,1}=H / \mathcal{B}_{1,1}$ and the orbits of $\left\langle\tau_{2}^{p}\right\rangle$ form a complete block system $\mathcal{E}$ of $K$. Then $K / \mathcal{E}$ has a Sylow $p$-subgroup of order $p^{3}$ that contains a regular elementary abelian subgroup and is imprimitive. By $[15$, Theorem 4] and [15, Lemma 6], we have that $K / \mathcal{B}_{1,1}$ is permutation isomorphic to a subgroup of $\operatorname{AGL}(1, p)$. Hence $H$ is permutation isomorphic to a subgroup of $\operatorname{AGL}(1, p)$ ₹ AGL $(2, p)$.

As $H \leq \operatorname{AGL}(1, p)$ 2 $\operatorname{AGL}(2, p)$, we have that $H$ normalizes $L=\left\langle\left.\tau_{1}\right|_{B},\left.\tau_{2}^{p}\right|_{B}: B \in\right.$ $\left.\mathcal{B}_{1,1}\right\rangle \cap H$. Also, $\langle L, P\rangle \leq \mathbb{Z}_{p} 2\left(\mathbb{Z}_{p} 2 \mathbb{Z}_{p}\right)$, a Sylow $p$-subgroup of $S_{p^{3}}$, so $L$ is contained in $P$, and so $L$ is contained in $\operatorname{fix}_{P}\left(\mathcal{B}_{1,1}\right)$. As $\left|\operatorname{Stab}_{P}(0,0)\right|=p$ and $\operatorname{Stab}_{P}(0,0)=\operatorname{Stab}_{\operatorname{fix}_{P}\left(\mathcal{B}_{1,1}\right)}(0,0)$ (as $P / \mathcal{B}_{1,1}$ has order $p$ and so is regular) also stabilizes only the points $(0, k p), k \in \mathbb{Z}_{p}$, $\operatorname{fix}_{P}\left(\mathcal{B}_{1,1}\right)$ contains $p^{2}$ distinct subgroups of order $p$ that are stabilizers of points, and as the identity is contained in all of them, these subgroups contain $p^{3}-\left(p^{2}-1\right)$ distinct elements. Thus $\operatorname{fix}_{P}(\mathcal{B})$ only contains $p^{2}-1$ nontrivial semiregular elements, and so $\left\langle\tau_{1}, \tau_{2}^{p}\right\rangle$ is the only semiregular elementary abelian subgroup of $\operatorname{fix}_{P}\left(\mathcal{B}_{1,1}\right)$. Also observe that if $h \in H$, then $h^{-1}\left\langle\tau_{1}, \tau_{2}^{p}\right\rangle h \leq L$ is a semiregular elementary abelian subgroup of $\operatorname{fix}_{P}\left(\mathcal{B}_{1,1}\right)$, and so is $\left\langle\tau_{1}, \tau_{2}^{p}\right\rangle$. Thus $\left\langle\tau_{1}, \tau_{2}^{p}\right\rangle \triangleleft H$.

Let $h \in H$. As $H / \mathcal{B}_{1,1} \leq \operatorname{AGL}(1, p)$, there exists $a \in \mathbb{Z}_{p}^{*}$ such that $\tau_{2}^{a} h^{-1} \tau_{2} h / \mathcal{B}_{1,1}=1$. As $\left\langle\tau_{1}, \tau_{2}^{p}\right\rangle \leq h^{-1}\left\langle\tau_{1}, \tau_{2}\right\rangle h$ we have that $\tau_{2}$ and $h^{-1} \tau_{2} h$ centralize $\left\langle\tau_{1}, \tau_{2}^{p}\right\rangle$. Thus $\tau_{2}^{a} h^{-1} \tau_{2} h$ centralizes $\left\langle\tau_{1}, \tau_{2}^{p}\right\rangle$. As a transitive abelian group is self-centralizing [6, Theorem 4.2A (v)], we have that $\left.\left.\tau_{2}^{a} h^{-1} \tau_{2} h\right|_{B} \in\left\langle\tau_{1}, \tau_{2}^{p}\right\rangle\right|_{B}$ for every $B \in \mathcal{B}_{1,1}$. Thus $\tau_{2}^{a} h^{-1} \tau_{2} h \in L$ and so $\tau_{2}^{a} h^{-1} \tau_{2} h \in P$. Finally, observe that the centralizer in $\operatorname{fix}_{P}\left(\mathcal{B}_{1,1}\right)$ of $\left\langle\tau_{1}, \tau_{2}^{p}\right\rangle$ is $\left\langle\tau_{1}, \tau_{2}^{p}\right\rangle$, and so $\tau_{2}^{a} h^{-1} \tau_{2} h \in\left\langle\tau_{1}, \tau_{2}^{p}\right\rangle$. Thus $h^{-1}\left\langle\tau_{1}, \tau_{2}\right\rangle h=\left\langle\tau_{1}, \tau_{2}\right\rangle$ and the result follows.

Lemma 30 Let $p \geq 5$ be prime, and $\gamma: \mathbb{Z}_{p} \times \mathbb{Z}_{p^{2}} \mapsto \mathbb{Z}_{p} \times \mathbb{Z}_{p^{2}}$ by $\gamma(i, j)=(i+$ $[a j(\bmod p)], j+i b p), a, b \in \mathbb{Z}_{p}^{*}$, and $P=\left\langle\tau_{1}, \tau_{2}, \gamma\right\rangle$ be of order $p^{4}$. If $H \leq S_{\mathbb{Z}_{p} \times \mathbb{Z}_{p^{2}}}$ with Sylow p-subgroup $P$, then $\left\langle\tau_{1}, \tau_{2}\right\rangle \triangleleft H$.

ProOF. In view of Lemma 29, we may assume without loss of generality that $H$ admits a complete block system $\mathcal{B}$ formed by the orbits of $\left\langle\tau_{2}^{p}\right\rangle$. Then $\left\langle\tau_{2}^{p}\right\rangle$ is a Sylow $p$-subgroup of $\operatorname{fix}_{H}(\mathcal{B})$ as $\left\langle\tau_{2}^{p}\right\rangle$ is a Sylow $p$-subgroup of $\operatorname{fix}_{P}(\mathcal{B})$. By [11, Lemma 4.2], one of the following is true:

i. $\operatorname{fix}_{H}(\mathcal{B})$ is cyclic and semiregular of order $p$,

ii. $H$ is permutation isomorphic to a subgroup of $S_{p^{2}} \times S_{p}$. Furthermore, there exists $J \leq S_{p^{2}}$ and $K \leq S_{p}$ such that $J \times K \triangleleft H$, or 
iii. $\operatorname{fix}_{H}(\mathcal{B})$ does not act faithfully on $B \in \mathcal{B}$ and a Sylow $p$-subgroup of $\operatorname{fix}_{H}(\mathcal{B})$ is not semiregular.

Note that (iii) cannot occur as a Sylow $p$-subgroup of $\operatorname{fix}_{H}(\mathcal{B})$ is $\left\langle\tau_{2}^{p}\right\rangle$, while $H$ cannot be permutation isomorphic to a subgroup of $S_{p^{2}} \times S_{p}$ as $P$ is not. Thus $\operatorname{fix}_{H}(\mathcal{B})$ is cyclic and semiregular of order $p$. Note that $H / \mathcal{B}$ is of degree $p^{2}$ and has Sylow $p$-subgroup of order $p^{3}$ with a transitive elementary abelian subgroup $\left\langle\tau_{1}, \tau_{2}\right\rangle / \mathcal{B}$. By $[15$, Theorem 4$]$, we have that $\left\langle\tau_{1}, \tau_{2}\right\rangle / \mathcal{B} \triangleleft H / \mathcal{B}$ as $p \geq 5$, and so $\operatorname{fix}_{H}(\mathcal{B})=\left\langle\tau_{2}^{p}\right\rangle$. Thus $\left\langle\tau_{1}, \tau_{2}\right\rangle \triangleleft H$ as required.

\section{Automorphism groups of Cayley digraphs of $\mathbb{Z}_{p} \times$ $\mathbb{Z}_{p^{2}}$}

The following result appears in [13, Lemma 28] with the additional hypothesis that $G$ contains a regular cyclic subgroup. This hypothesis was essentially not used in the proof of [13, Lemma 28], and we have the following result.

Lemma 31 Let $G \leq S_{m k}$ be 2-closed. If $G$ admits a genuine nontrivial complete block system $\mathcal{B}$ consisting of $m$ blocks of size $k$ such that $\left.\operatorname{fix}_{G}(\mathcal{B})\right|_{B}$ is primitive and $\operatorname{fix}_{G}(\mathcal{B})$ does not act faithfully on $B \in \mathcal{B}$, then $G=G_{1} \cap G_{2}$, where $G_{1}=S_{r} \imath H_{1}$ and $G_{2}=H_{2} \imath S_{k}, H_{1}$ is a 2-closed group of degree $m k / r, H_{2}$ is a 2-closed group of order $m$, and $r \mid m$.

We now prove the main result of this paper, and note that in the statement of this result, $\alpha_{2}$ and $\bar{a}$ are as defined in Definition 24 .

Theorem 32 Let $p \geq 5$ be prime, and $H \leq S_{\mathbb{Z}_{p} \times \mathbb{Z}_{p^{2}}}$ be a 2-closed group that contains the left regular representation of $\mathbb{Z}_{p} \times \mathbb{Z}_{p^{2}}$. Then one of the following is true for some $\alpha_{1} \in \operatorname{Aut}\left(\mathbb{Z}_{p} \times \mathbb{Z}_{p^{2}}\right), A \leq \operatorname{Aut}\left(\mathbb{Z}_{p} \times \mathbb{Z}_{p^{2}}\right)$ of order relatively prime to $p, D \leq \mathbb{Z}_{p}^{*}$, and $E \leq \mathbb{Z}_{p^{2}}^{*}$ of order relatively prime to $p$ :

1. $H=S_{\mathbb{Z}_{p} \times \mathbb{Z}_{p^{2}}}$,

2. $\left(\mathbb{Z}_{p} \times \mathbb{Z}_{p^{2}}\right)_{L} \triangleleft H$,

3. $\alpha_{1}^{-1} H \alpha_{1}=S_{p} \times B$, where $B \leq N\left(p^{2}\right)$ is 2-closed of order dividing $(p-1) p^{2}$ and so has a cyclic Sylow p-subgroup,

4. $H$ is permutation isomorphic to $X \times S_{p^{2}}$ or $C((X \succ Y) \times Z)$, where $X, Y, Z \leq S_{p}$ are 2-closed and $C \leq \operatorname{Aut}\left(\mathbb{Z}_{p}^{3}\right)$.

5. $\alpha_{1}^{-1} H \alpha_{1}=H_{1}$ 々 $H_{2}$ or $H_{2}$ 々 $H_{1}$ where $H_{1}$ is a 2-closed group of degree $p$ and $H_{2}$ is a 2-closed group of degree $p^{2}$,

6. $\alpha_{1}^{-1} H \alpha_{1}=\left\{(i, j) \mapsto\left(\omega(i), \alpha j+a+p b_{i}\right): \omega \in S_{p}, \alpha \in E, a \in \mathbb{Z}_{p^{2}}, b_{i} \in \mathbb{Z}_{p}\right\}$,

7. $\alpha_{1}^{-1} H \alpha_{1}=A \cdot P$, where $P=\left\langle\tau_{1}, \tau_{2},\left.\tau_{2}^{p}\right|_{B}: B \in \mathcal{B}_{2}\right\rangle$, 
8. $\alpha_{2}^{-c} \alpha_{1} H \alpha_{1}^{-1} \alpha_{2}^{c}=\left\{(i, j) \mapsto\left(\omega_{j}(\bmod p)(i), \alpha j+b\right): \omega_{j}(\bmod p) \in S_{p}, \alpha \in E, b \in \mathbb{Z}_{p^{2}}\right\}$, $c=0,1$, and $\alpha_{1}=\bar{a}$ for some $a \in \mathbb{Z}_{p}^{*}$, or

9. $\alpha_{2}^{-c} \alpha_{1}^{-1} H \alpha_{1} \alpha_{2}^{c}=A \cdot\left\{(i, j) \mapsto\left(d_{j}(\bmod p) i+c_{j}(\bmod p), j+b\right): d_{j(\bmod p)} \in D\right.$, $c_{j(\bmod p)} \in \mathbb{Z}_{p}$, and $\left.b \in \mathbb{Z}_{p^{2}}\right\}$, for $c=0,1$, and $\alpha_{1}=\bar{a}$ for some $a \in \mathbb{Z}_{p}^{*}$.

Proof. Let $P$ be a Sylow $p$-subgroup of $H$ that contains $\left\langle\tau_{1}, \tau_{2}\right\rangle$. By Theorem 12, there exists $\alpha_{1} \in \operatorname{Aut}\left(\mathbb{Z}_{p} \times \mathbb{Z}_{p^{2}}\right)$ such that one of the following is true:

(i) $H=S_{\mathbb{Z}_{p} \times \mathbb{Z}_{p^{2}}}$,

(ii) $P=\left(\mathbb{Z}_{p} \times \mathbb{Z}_{p^{2}}\right)_{L}$,

(iii) $P=\alpha_{1}^{-1}\left\langle\tau_{1}, \tau_{2},\left.\tau_{2}^{p}\right|_{B}: B \in \mathcal{B}_{1,1}\right\rangle \alpha_{1} \cong \mathbb{Z}_{p} \times\left(\mathbb{Z}_{p}\left\langle\mathbb{Z}_{p}\right)\right.$,

(iv) $P=\alpha_{1}^{-1}\left\langle\tau_{1}, \tau_{2},\left.\tau_{2}^{p}\right|_{B}: B \in \mathcal{B}_{2}\right\rangle \alpha_{1}$,

(v) $P=\alpha_{1}^{-1}\left\langle\tau_{1}, \tau_{2},\left.\tau_{1}\right|_{B}: B \in \mathcal{B}_{1,1}\right\rangle \alpha_{1}$,

(vi) if $\gamma: \mathbb{Z}_{p} \times \mathbb{Z}_{p^{2}} \mapsto \mathbb{Z}_{p} \times \mathbb{Z}_{p^{2}}$ by $\gamma(i, j)=(i+[a j(\bmod p)], j+i b p), a, b \in \mathbb{Z}_{p}^{*}$, then $P=\alpha_{1}^{-1}\left\langle\tau_{1}, \tau_{2}, \gamma\right\rangle \alpha_{1}$, and $|P|=p^{4}$,

(vii) $\alpha_{1}^{-1} P \alpha_{1}=P_{1} \prec P_{2}$, where $P_{1}$ is 2-closed $p$-group of degree $p^{2}$ and contains a regular subgroup isomorphic to $\mathbb{Z}_{p^{2}}$ or $\mathbb{Z}_{p}^{2}$, and $P_{2} \leq S_{p}$ is cyclic of order $p$,

(viii) $\alpha_{1}^{-1} P \alpha_{1}=P_{1} \prec P_{2}$, where $P_{2} \leq S_{p}$ is cyclic of order $p$, and $P_{1} \leq S_{p^{2}}$ is 2-closed $p$-subgroup of degree $p^{2}$ and contains a regular subgroup isomorphic to $\mathbb{Z}_{p^{2}}$.

If (i) occurs, then (1) occurs. If (ii) occurs, then by Theorem 13, either (2) occurs or $H$ is permutation isomorphic to $S_{p} \times B$, where $B \leq N\left(p^{2}\right)$ has order dividing $(p-1) p^{2}$, and so has a cyclic Sylow $p$-subgroup. Applying Lemma 11, we see that $H$ is also 2-closed. Note that $H$ admits orthogonal complete block systems $\mathcal{B}$ and $\mathcal{C}$ consisting of $p$ blocks of size $p^{2}$ and $p$ blocks of size $p$ formed by the orbits of the subgroups of $H$ permutation isomorphic to $1_{S_{p}} \times B$ and $S_{p} \times 1_{S_{p^{2}}}$, respectively. As $\mathcal{B}$ and $\mathcal{C}$ are genuine, $\mathcal{B}$ is formed by the orbits of $\left\langle\tau_{2} \tau_{1}^{a}\right\rangle$ while $\mathcal{C}$ is formed by the orbits of $\left\langle\tau_{1} \tau_{2}^{b p}\right\rangle, a, b \in \mathbb{Z}_{p}$. Let $\alpha_{1} \in \operatorname{Aut}\left(\mathbb{Z}_{p} \times \mathbb{Z}_{p^{2}}\right)$ such that $\alpha_{1}^{-1}\left\langle\tau_{2} \tau_{1}^{a}\right\rangle \alpha_{1}=\left\langle\tau_{2}\right\rangle$ and $\alpha_{1}^{-1}\left\langle\tau_{2} \tau_{2}^{b p}\right\rangle \alpha_{1}=\left\langle\tau_{1}\right\rangle$. Such an $\alpha_{1}$ exists by [5, pg. 5]. Then $\alpha_{1}^{-1} H \alpha_{1}=S_{p} \times B$ and (3) occurs. If (iii) occurs, then (4) follows by Lemma 14 .

If (iv) occurs, then by Lemma 20, we have that $\alpha_{1}^{-1} H \alpha_{1}=K \leq\{(i, j) \mapsto(\omega(i), \alpha j+$ $\left.\left.a+p b_{i}\right): \omega \in S_{p}, \alpha \in \mathbb{Z}_{p^{2}}^{*}, a, b_{i} \in \mathbb{Z}_{p^{2}}\right\}$. Then $K$ admits $\mathcal{B}_{2}$ as a complete block system. If $K / \mathcal{B}_{2} \leq \operatorname{AGL}(1, p)$, then (7) occurs by Lemma 21. If $K / \mathcal{B}_{2} \not \leq \operatorname{AGL}(1, p)$, then by Theorem 6 , we have that $K / \mathcal{B}_{2}$ is doubly-transitive with nonabelian simple socle. Let $L$ be the normal closure of $\left\langle\tau_{1}, \tau_{2}\right\rangle$ in $K$. As $K / \mathcal{B}_{2}$ is a doubly-transitive group with nonabelian simple socle, we have that $L / \mathcal{B}_{2}$ is a nonabelian simple group, and so by Theorem $6, L / \mathcal{B}$ is doubly-transitive. By the definition of $K$, we have that $\left.\operatorname{fix}_{L}\left(\mathcal{B}_{2}\right)\right|_{B_{2}} \cong$ $\mathbb{Z}_{p^{2}}$ for every $B_{2} \in \mathcal{B}_{2}$. Then $\operatorname{fix}_{L}\left(\mathcal{B}_{2}\right) \leq\left\{(i, j) \mapsto\left(i, j+a+b_{i} p\right): a, b_{i} \in \mathbb{Z}_{p^{2}}\right\}$. As $\left\langle\left.\tau_{2}^{p}\right|_{B_{2}}: B_{2} \in \mathcal{B}_{2}\right\rangle=\left\{(i, j) \mapsto\left(i, j+b_{i} p\right): b \in \mathbb{Z}_{p^{2}}\right\}$, we conclude that $K$ contains a 
subgroup $M$ such that $M / \mathcal{B}_{2}=L / \mathcal{B}_{2}$ and $\operatorname{fix}_{M}\left(\mathcal{B}_{2}\right)=\mathbb{Z}_{p^{2}}$. By [11, Corollary 6.5], we have that $M=T \times \mathbb{Z}_{p^{2}}$, there $T \leq S_{p}$ is a doubly-transitive nonabelian group. Then $M^{(2)}=S_{p} \times \mathbb{Z}_{p^{2}}$ by Lemma 11 and the map $(i, j) \mapsto(\omega(i), j)$ is in $K$ for every $\omega \in S_{p}$. Thus if $(i, j) \mapsto\left(\omega(i), \alpha j+a+p b_{i}\right) \in K$, then the map $(i, j) \mapsto(i, \alpha j) \in K$. Letting $E \leq \mathbb{Z}_{p^{2}}^{*}$ such that $\left.\operatorname{fix}_{K}\left(\mathcal{B}_{2}\right)\right|_{B_{2}}=E \cdot\left(\mathbb{Z}_{p^{2}}\right)_{L}$ for some $B_{2} \in \mathcal{B}_{2}$, (6) holds.

If (v) occurs, then by Lemma 25 for $c=0$ or 1 , and $a \in \mathbb{Z}_{p}^{*}, K=\alpha_{2}^{-c} \bar{a}^{-1} H \bar{a} \alpha_{2}^{c} \leq$ $\left\{(i, j) \mapsto\left(\beta_{j(\bmod p)}(i), \alpha j+b\right): \beta_{j(\bmod p)} \in S_{p}, \alpha \in \mathbb{Z}_{p^{2}}^{*}, b \in \mathbb{Z}_{p^{2}}\right\}$. Then $K$ admits a complete block system $\mathcal{B}$ formed by the orbits of $\left\langle\tau_{1}\right\rangle$. If $\left.\operatorname{fix}_{K}(\mathcal{B})\right|_{B} \leq \operatorname{AGL}(1, p)$ for every $B \in \mathcal{B}$, then $K \leq\left\{(i, j) \mapsto\left(\beta_{j}(\bmod p)(i), \alpha j+b\right): \beta_{j}(\bmod p) \in \operatorname{AGL}(1, p), \alpha \in\right.$ $\left.\mathbb{Z}_{p^{2}}^{*}, b \in \mathbb{Z}_{p^{2}}\right\}$. Then (9) follows from Lemma 26. Otherwise, fix $\left.x_{K}(\mathcal{B})\right|_{B}$ is a doublytransitive group with nonabelian simple socle $T$ by Theorem 6 . Define an equivalence relation $\equiv$ on the blocks of $\mathcal{B}$ by $B \equiv B^{\prime}$ if and only if whenever $k \in \operatorname{fix}_{K}(\mathcal{B})$ then $\left.k\right|_{B}$ is a $p$-cycle if and only if $\left.k\right|_{B^{\prime}}$ is also a $p$-cycle. Clearly the equivalence classes of $\equiv$ are $\mathcal{B}_{1,1}$ as $\left\langle\left.\tau_{1}\right|_{B_{1,1}}: B_{1,1} \in \mathcal{B}_{1,1}\right\rangle$ is a Sylow $p$-subgroup of $\operatorname{fix}_{H}(\mathcal{B})$. By Lemma 10 we have that if $k \in \operatorname{fix}_{K}(\mathcal{B})$, then $\left.k\right|_{B_{1,1}} \in \operatorname{fix}_{K}(\mathcal{B})$ for every $B_{1,1} \in \mathcal{B}_{1,1}$. We conclude that $\left\{(i, j) \mapsto\left(t_{j(\bmod p)}(i), j\right): t_{j(\bmod p)} \in T\right\} \leq K$. Then $L=\{(i, j) \mapsto(t(i), j+b): t \in$ $\left.T, b \in \mathbb{Z}_{p^{2}}\right\} \leq K$, and $L=T \times \mathbb{Z}_{p^{2}}$. By Lemma 11, $L^{(2)}=T^{(2)} \times\left(\mathbb{Z}_{p^{2}}\right)^{(2)}=S_{p} \times \mathbb{Z}_{p^{2}} \leq K$ and so if $(i, j) \mapsto\left(\beta_{j}(\bmod p)(i), \alpha j+b\right) \in K$, then the map $(i, j) \mapsto(i, \alpha j) \in K$. Letting $E \leq \mathbb{Z}_{p^{2}}^{*}$ such that $K / \mathcal{B}=E \cdot\left(\mathbb{Z}_{p^{2}}\right)_{L},(8)$ occurs.

If (vi) occurs, then (2) occurs by Lemma 30. If (vii) or (viii) occur, then let $D$ be a color digraph such that $\operatorname{Aut}(D)=H$. Then $D$ can be written as a nontrivial wreath product as $P$ is a nontrivial wreath product. We conclude that $H$ is a nontrivial wreath product by [14, Theorem 5.7], and so (5) occurs.

Acknowledgement: The author is indebted to the anonymous referee for comments and suggestions which made the paper both shorter and easier to read.

\section{References}

[1] B. Alspach, Point-symmetric graphs and digraphs of prime order and transitive permutation groups of prime degree, J. Combinatorial Theory Ser. B 15 (1973), 12-17.

[2] W. Burnside, On some properties of groups of odd order, J. London Math. Soc. 33 (1901), 162-185.

[3] P. J. Cameron, Finite permutation groups and finite simple groups, Bull. London Math. Soc. 13 (1981), no. 1, 1-22.

[4] P. J. Cameron, M. Giudici, G. A. Jones, W. M. Kantor, M. H. Klin, D. Marušič, and L. A. Nowitz, Transitive permutation groups without semiregular subgroups, J. London Math. Soc. (2) 66 (2002), no. 2, 325-333.

[5] H. S. M. Coxeter and W. O. J. Moser, Generators and relations for discrete groups, Second edition. Ergebnisse der Mathematik und ihrer Grenzgebiete, Neue Folge, Band 14, Springer-Verlag, Berlin, 1965. 
[6] J. D. Dixon and B. Mortimer, Permutation groups, Graduate Texts in Mathematics, vol. 163, Springer-Verlag, New York, 1996.

[7] E. Dobson, Isomorphism problem for Cayley graphs of $\mathbb{Z}_{p}^{3}$, Discrete Math. 147 (1995), no. 1-3, 87-94.

[8] E. Dobson, Classification of vertex-transitive graphs of order a prime cubed. I, Discrete Math. 224 (2000), no. 1-3, 99-106.

[9] E. Dobson, On groups of odd prime-power degree that contain a full cycle, Discrete Math. 299 (2005), no. 1-3, 65-78.

[10] E. Dobson, Automorphism groups of metacirculant graphs of order a product of two distinct primes, Combin. Probab. Comput. 15 (2006), no. 1-2, 105-130.

[11] E. Dobson, On overgroups of regular abelian p-groups, Ars Math. Contemp. 2 (2009), no. $1,59-76$.

[12] E. Dobson and I. Kovács, Automorphism groups of Cayley digraphs of $\mathbb{Z}_{p}^{3}$, Electron. J. Combin. 16 (2009), no. 1, Research Paper 149, 20.

[13] E. Dobson and J. Morris, On automorphism groups of circulant digraphs of squarefree order, Discrete Math. 299 (2005), no. 1-3, 79-98.

[14] E. Dobson and J. Morris, Automorphism groups of wreath product digraphs, Electron. J. Combin. 16 (2009), no. 1, Research Paper 17, 30.

[15] E. Dobson and D. Witte, Transitive permutation groups of prime-squared degree, J. Algebraic Combin. 16 (2002), no. 1, 43-69.

[16] G. A. Jones, Cyclic regular subgroups of primitive permutation groups, J. Group Theory 5 (2002), no. 4, 403-407.

[17] L. A. Kalužnin and M. H. Klin, Some numerical invariants of permutation groups, Latviǔsk. Mat. Ežegodnik (1976), no. Vyp. 18, 81-99, 222.

[18] M. H. Klin and R. Pöschel, The König problem, the isomorphism problem for cyclic graphs and the method of Schur rings, Algebraic methods in graph theory, Vol. I, II (Szeged, 1978), Colloq. Math. Soc. János Bolyai, vol. 25, North-Holland, Amsterdam, 1981, pp. 405-434.

[19] C. H. Li, The finite primitive permutation groups containing an abelian regular subgroup, Proc. London Math. Soc. (3) 87 (2003), no. 3, 725-747.

[20] C. H. Li, Permutation groups with a cyclic regular subgroup and arc transitive circulants, J. Algebraic Combin. 21 (2005), no. 2, 131-136.

[21] J. D. P. Meldrum, Wreath products of groups and semigroups, Pitman Monographs and Surveys in Pure and Applied Mathematics, vol. 74, Longman, Harlow, 1995.

[22] I. N. Ponomarenko, Determination of the automorphism group of a circulant association scheme in polynomial time, Zap. Nauchn. Sem. S.-Peterburg. Otdel. Mat. Inst. Steklov. (POMI) 321 (2005), Vopr. Teor. Predst. Algebr. i Grupp. 12, 251-267, 301.

[23] G. Sabidussi, Vertex-transitive graphs, Monatsh. Math. 68 (1964), 426-438.

[24] H. Wielandt, Finite permutation groups, Translated from the German by R. Bercov, Academic Press, New York, 1964. 